TROISIÈME PARTIE

\title{
Secteurs de recherche à développer
}



La troisième partie du rapport est consacrée aux secteurs de recherches de nature disciplinaire à approfondir en vue d'une meilleure connaissance du fonctionnement biogéochimique des terres émergées. Les deux domaines retenus l'ont été en partant d'une constatation fondamentale, à savoir que la biogéochimie continentale repose avant tout sur le fonctionnement du sol, celui-ci constituant assurément le pilier central de l'écosphère terrestre. Or, l'étude de l'activité d'un objet naturel aussi complexe est particulièrement ardue, en raison des interactions existant en permanence entre les principaux constituants, à savoir : constituants minéraux - matières organiques - microflore tellurique; les deux derniers étant notamment les acłeurs majeurs du comportement biogéochimique de la surface de la planète sur lesquels il convient plus particulièrement d'insister dans ce document. Ce sont donc eux qui feront l'objet des chapitres 7 et 8 .

Le chapitre 7 est consacré à l'étude des micro-organismes des sols, vaste domaine encore trop peu connu, alors qu'il a été dit souvent que le monde leur appartenait.

Le chapitre 8 porte sur les matières organiques des sols (MOS), qui sont à la base du cycle des bio-éléments, tels le carbone et surtout l'azote, en relation notamment avec la production biologique d'azote nitrique indispensable à la nutrition minérale des végétaux. 

CHAPITRE 7

\section{Les micro-organismes, clé des recyclages biogéochimiques}

JACQUES BERTHELIN 



\section{Introduction - Rappel de quelques données}

Depuis que les premiers organismes vivants, avec les ancêtres des actuelles bactéries, sont apparus sur Terre, voilà environ 3,5 milliards d'années à la suite de phénomènes qui se sont déroulés sur quelques centaines de millions $d$ 'années (Bada, 2004), les micro-organismes ou protistes ont été amenés à coloniser tous les compartiments de l'enveloppe terrestre. Cela concerne notamment les deux grands compartiments où la vie est particulièrement active, à savoir les sols et les océans incluant les eaux continentales et les sédiments récents, qui renferment l'ensemble des grands groupes microbiens (bactéries, champignons, algues, protozocires). Ils s'y sont développés, plus particulièrement les communautés bactériennes, en mettant en œuvre des stratégies énergétiques et nutritionnelles très diversifiées, rappelées schématiquement dans la figure 7.1 ( $d^{\prime}$ 'après Berthelin, 1988). Ces communautés microbiennes sont en effet très abondantes $\left(1 \mathrm{~g}\right.$ de sol contient dans les horizons de surface $10^{6}$ à $10^{9}$ bactéries), ubiquistes et à grande diversité fonctionnelle : elles peuvent vivre tant en milieu minéral qu'en milieu organique et en présence ou absence d'oxygène, qu'en milieu très acide ou très alcalin, en conditions de faibles ou fortes salinités ou bien encore en milieux particulièrement froids ou très chauds (Madigan et al., 2000). Tout cela se rapporte avant tout aux milieux superficiels de la planète, domaine fondamental de la biosphère, et c'est dans ce cadre que vont être rappelées une série de données concernant le fonctionnement général des systèmes microbiens, en particulier des micro-organismes non pathogènes.

Au demeurant, il est bon de signaler la découverte récente d'une « biosphère souterraine profonde » dont il sera fait état brièvement à la fin de ce chapitre.

1. D'un point de vue énergétique et nutritionnel, on peut globalement distinguer quatre grands domaines d'activités microbiennes, en fonction des sources de carbone et d'énergie utilisées:

- les organismes photolithotrophes, protistes supérieurs à structure eucaryotique (algues) et protistes inférieurs à structure procaryotique (cyanobactéries), utilisant l'énergie électromagnétique (lumineuse) et le carbone inorganique comme source de carbone. Ce sont avant tout des organismes producteurs de biomasse et de matières organiques. Certains d'entre eux interviennent dans la fixation d'azote moléculaire comme les cyanobactéries qui seraient responsables des deux tiers de la fixation non symbiotique de l'azote de par leur présence massive dans les océans, dans l'absorption-accumulation d'éléments minéraux et la néoformation de minéraux (par exemple, diatomées). Ils permettent et entretiennent la vie hétérotrophe dans les milieux extrêmes (désertiques, montagnards) et 
Oxydation de composés minéraux et organiques source d'énergie

Réduction de composés accepteurs d'électrons :

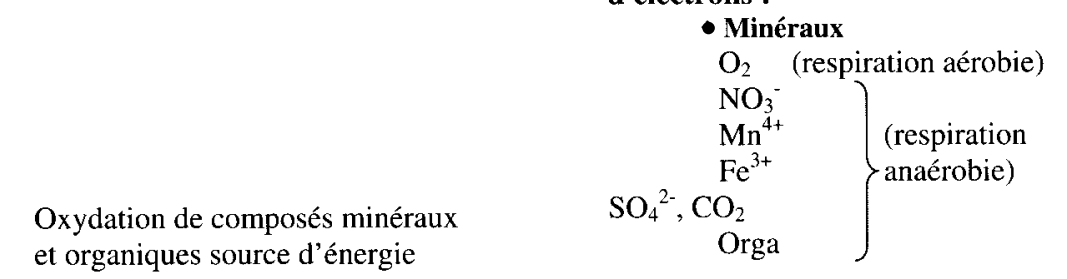
(donneurs d'électrons)

- Oganiques : fermentations*

\section{Absorption de nutriments organiques et minéraux $(\mathrm{C}, \mathrm{N}, \mathrm{Fe}, \mathrm{S}, \ldots)$}

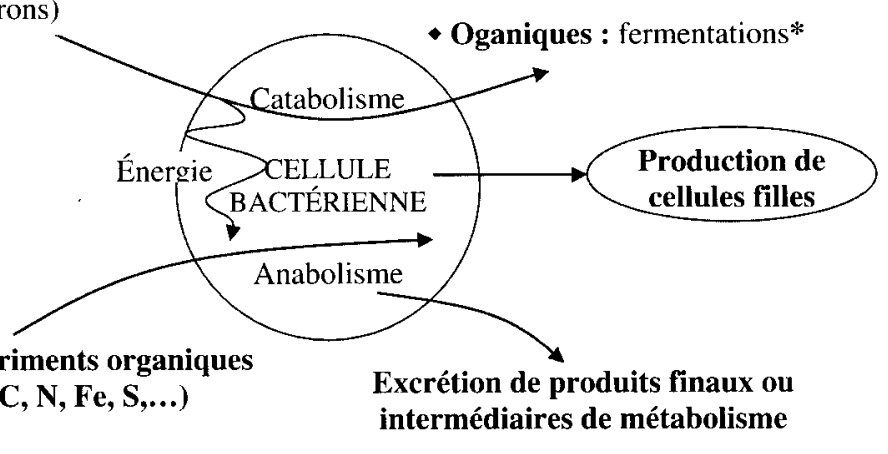

* Composés organiques issus de leur métabolisme.

\section{Figure 7.1}

Schéma simplifié du fonctionnement des bactéries chimiolithotrophes et chimio-organotrophes. (D'après Berthelin, 1998).

forment avec d'autres micro-organismes des associations pionnières (par exemple, association algues-champignons : les lichens);

- les micro-organismes photo-organotrophes, en fait des bactéries (utilisant une source d'énergie électromagnétique, c'est-à-dire lumière, et des sources de carbone organiques), représentent un groupe modeste (bactéries dites «non sulfureuses pourpres》) (par exemple, Rhodospirillum) d'un grand intérêt écologique et fonctionnel, mais dont l'impact sur le fonctionnement des cycles est limité, sauf dans certains écosystèmes marins et lacustres;

- les micro-organismes qui utilisent des sources d'énergie organiques et les matières organiques comme source de carbone et d'énergie (organismes chimio-organotrophes c'est-à-dire hétérotrophes) comprennent l'ensemble des champignons et la plus grande partie des bactéries. Ils sont considérés comme des décomposeurs qui assurent la biodégradation et minéralisation des composés organiques naturels ou xénobiotiques, mais ont aussi des fonctions importantes de producteurs : production de nutriments au cours de la minéralisation des matières organiques, production de substances humiques, production de métabolites d'intérêt 
environnemental ou pharmaceutique, ou agronomique ou alimentaire... et sont impliqués dans les phénomènes de transformation (dissolutionaltération; absorption-accumulation-néoformation) de minéraux et de composés organominéraux;

- enfin, un quatrième groupe correspond aux organismes chimiolithotrophes qui utiliseront des matières minérales réduites (ammonium, fer ferreux, soufre réduit des sulfures, ... ) comme source d'énergie et le carbone inorganique $\left(\mathrm{CO}_{2}\right)$ comme source de carbone. Ce sont des groupes fonctionnels d'un intérêt très fondamental pour le fonctionnement de nombreux cycles (fer, azote, soufre. . .).

2. Les micro-organismes du sol, des eaux douces, des sédiments et des océans peuvent vivre en présence ou en absence $d^{\prime}$ 'oxygène. Ils possèdent des systèmes de respiration aérobie (oxygène accepteur d'électrons), et c'est le cas de pratiquement tous les champignons et d'un grand nombre de bactéries. D'autres populations peuvent vivre en absence et en présence d'oxygène : ce sont des organismes aéro-anaérobies. Enfin un grand nombre de communautés bactériennes vivent en absence totale d'oxygène. Ce sont les bactéries anaérobies strictes. Ces bactéries disposent de système de respiration anaérobie et utilisent des accepteurs d'électrons minéraux autres que l'oxygène (nitrate, fer ferrique, manganèse manganique, sulfate, dioxyde de carbone et, pour quelques-unes, des accepteurs organiques par exemple, fumarate).

Certaines bactéries et quelques champignons (par exemple les levures) utilisent comme accepteurs d'électrons des produits organiques issus de leur catabolisme (qu'ils produisent eux-mêmes). Leur métabolisme est dit « fermentaire ». Ces fermentations se déroulent en conditions anoxiques.

3. Ces diverses communautés microbiennes jouent un rôle majeur dans un grand nombre de domaines, qu'il est utile de rappeler :

- la biodégradation et minéralisation des matières organiques et la production de nutriments. Elles sont fondamentalement impliquées dans la production des formes assimilables de l'azote, du phosphore, du soufre, du fer... et la libération d'éléments nutritifs (potassium, calcium, magnésium...);

- les réactions d'oxydoréduction, de méthylation, de déméthylation qui déterminent directement les changements d'état de nombreux éléments majeurs ou en traces et de radionucléides (carbone, azote, soufre, fer, manganèse... mercure, sélénium, chrome, arsenic);

- le fonctionnement des cycles biogéochimiques: C, N, P, S, Fe... dont elles régulent et déterminent même totalement certaines étapes; 
- le couplage de cycles biogéochimiques (par exemple, $\mathrm{C}$ et $\mathrm{N} ; \mathrm{C}$ et $\mathrm{Fe}$; $\mathrm{C}-\mathrm{Fe}-\mathrm{S}$ et éléments en traces associés...);

- la production de gaz à effet de serre $\left(\mathrm{CO}_{2}, \mathrm{CH}_{4}, \mathrm{~N}_{2} \mathrm{O}\right.$. . ) et la qualité de l'atmosphère;

- I'altération et la néoformation de minéraux;

- les changements de conditions acido-basiques et oxydoréductrices du milieu;

- la formation d'associations bénéfiques (ou non) avec les racines des plantes, la faune du sol : associations rhizosphériques symbiotiques ou non symbiotiques améliorant la nutrition végétale. .., associations synergiques avec les divers animaux du sol : lombrics, termites, collemboles améliorant l'humification, la structure du sol. . (Lavelle et Spain 2001);

- la qualité des eaux : épuration par biodégradation des composés organiques $d^{\prime}$ origine naturelle ou anthropique ou par biosorption ou bioaccumulation de métaux, ... mais aussi dégradation par acidification des eaux par production d'acides (par exemple, nitrique, sulfurique...);

- la qualité et le rendement des productions végétales;

- le traitement de pollutions organiques et minérales, de déchets...;

- le traitement de matières premières (extraction-accumulation de métaux, élimination de substances indésirables. . .);

- la formation de dépôts minéraux (gisements).

4. Les principaux processus microbiens et les grands groupes fonctionnels sont relativement bien connus, mais la composition des communautés impliquées, leur dynamique, les mécanismes géomicrobiologiques et les paramètres physiques, chimiques, biologiques qui les déterminent sont mal définis.

Par ailleurs, on estime que seulement $1 \%$ des populations bactériennes du sol peuvent être cultivées au laboratoire par les méthodes actuelles sans savoir si ces organismes cultivables sont représentatifs des populations bactériennes (Kirk et al., 2004). Cette situation est d'autant plus surprenante que la ressource génétique dans les sols est évaluée à 4000 unités génomiques bactériennes différentes par gramme de sol (Torsvik et al., 1990) et peut-être plus. Il en est de même pour les champignons (van Elsas et al., 2000). À titre d'exemple particulièrement significatif, on ne sait toujours pas étudier en culture pure (hors symbiose avec les racines) les champignons endomycorhiziens (mycorhizes à arbuscules) qui forment des associations symbiotiques avec environ $80 \%$ des 
espèces végétales et sont bénéfiques pour la nutrition et la protection des plantes concernées.

Dans ce rapport, il y a lieu d'insister sur ce rôle majeur des micro-organismes, dans tous les milieux de l'enveloppe terrestre, pour souligner l'intérêt de développer les connaissances en écologie microbienne, biogéochimie microbienne et géomicrobiologie. Ces objectifs concernent les processus microbiens et les organismes impliqués, les paramètres «écologiques : physiques, chimiques, biologiques » qui déterminent leur activité, et enfin les " retombées environnementales » et « socio-économiques» qui en résultent. Les principaux aspects vont être présentés maintenant.

\section{Production et acquisition de nutriments}

Les micro-organismes (bactéries et champignons) sont les agents essentiels de la production de nutriments pour les micro-organismes eux-mêmes et les végétaux. En biodégradant et minéralisant les matières organiques et en produisant, selon les conditions de milieux, $\mathrm{CO}_{2}, \mathrm{CH}_{4}, \mathrm{NH}_{4}, \mathrm{NO}_{3}, \mathrm{SO}_{4}, \mathrm{H}_{2} \mathrm{~S}, \mathrm{HPO}_{4}, \mathrm{H}_{2}$ $\mathrm{PO}_{4} \ldots$, ils mettent à la disposition d'eux-mêmes et des plantes, les éléments nécessaires à leur nutrition phosphorée, azotée, soufrée... qu'ils complètent en contribuant à la libération d'éléments majeurs (calcium, magnésium, potassium, ...) et d'oligo-éléments (fer, manganèse, cuivre...) restitués par les résidus (comme les litières) et par les débris végétaux et microbiens. Dans les cycles biogéochimiques très localisés, qui se déroulent dans une "boucle " "plantessols-micro-organismes", ils assurent le recyclage des éléments nutritifs sur des échelles de temps plus ou moins courtes (des milieux tropicaux à cycles rapides aux milieux tempérés ou montagnards ou boréaux, à cycles plus longs).

Les micro-organismes sont aussi des agents de l'altération efficace des minéraux (Berthelin, 1988) et assurent soit en étant étroitement associés aux racines (milieux rhizosphériques) ou indépendamment du système racinaire, la libération d'autres nutriments à partir des minéraux et constituants organominéraux des sols (K, Mg, Fe, Mn. ..) (Leyval et Berthelin, 1991...).

Ces deux grandes voies d'acquisition des nutriments, minéralisation des produits végétaux et altération des minéraux, sont dominantes dans les milieux peu ou pas fertilisés (écosystèmes forestiers...), mais elles interviennent sans aucun doute pour mobiliser efficacement certains éléments (phosphore, fer, ...) dans les agrosystèmes.

Dans les cycles biogéochimiques, le rôle des micro-organismes est mal distingué des processus physicochimiques. Les formes sous lesquelles les 
éléments sont disponibles (organominérales ou minérales) et sont mobilisés et recyclés sont à préciser, ainsi que les paramètres majeurs impliqués Ivégétation, roche mère, état hydrique du sol....).

\section{2 | Rôle majeur des micro-organismes dans les étapes fondamentales du cycle de divers éléments}

Les micro-organismes du sol, des eaux, des sédiments, des océans (bactéries et champignons) interviennent comme agents principaux du fonctionnement du cycle biogéochimique de divers éléments majeurs et en traces (carbone, azote, soufre, phosphore, fer, mercure, sélénium, arsenic, ...) (Dommergues et Mangenot, 1970; Madigan et al., 2000 ; Ehrlich ; 2002 ; Gobat et al., 2003). Il paraît nécessaire de rappeler, en complément des présentations faites dans la première partie de ce rapport et avec une approche géomicrobiologique et fonctionnelle, I'implication des micro-organismes dans le fonctionnement du cycle de certains éléments et $d^{\prime}$ indiquer seulement leur intervention plus ou moins significative dans d'autres.

\subsection{Cycle du carbone}

Tout d'abord pour le cycle du carbone, cycle clé du fonctionnement de l'écosystème terrestre (cf. chapitre 1), ils assurent des fonctions essentielles en tant que décomposeurs (biotransformation-biodégradation-minéralisation des matières organiques), mais aussi de producteurs de matières organiques (biomasse, composés humiques) et de diverses substances d'intérêt pour le fonctionnement des écosystèmes ou pour des applications industrielles, pharmaceutiques (par exemple, production d'antibiotiques, de vitamines, de surfactants...). On peut distinguer d'un point de vue d'écologie fonctionnelle globale, des phases et boucles aérobies et anaérobies de fixation (réduction du $\mathrm{CO}_{2}$ et production de matières organiques) et de minéralisation (biodégradation et production de $\mathrm{CO}_{2}$ ) du cycle de carbone. Un cycle concernant le méthane (méthanogenèse, méthanotrophie) peut être distingué. Le cycle actuel du carbone peut paraître relativement bien connu, mais l'ensemble des organismes qui interviennent et les paramètres de contrôle de leur activité sont encore à préciser (par exemple, conditions de stabilisation-minéralisation du carbone, facteurs déterminant la production de méthane et plus généralement de gaz à effet de serre, ...). Par ailleurs, des applications très ciblées concernent la production de composés organiques d'intérêt agricole, forestier, pharmaceutique... et la biodégradation de polluants.

Divers aspects du cycle du carbone sont traités par ailleurs, aux chapitres 1 et 8 auxquels on peut se reporter. 


\subsection{Cycle de l'azote}

Dans le cas du cycle biogéochimique de l'azote qui présente des similitudes avec celui du soufre (cf. chapitre 2) et est couplé étroitement au cycle du carbone, on peut rappeler les grandes étapes pour souligner leur spécificité d'une part et leurs relations avec d'autres cycles d'autre part.

- La fixation de l'ázote moléculaire de l'atmosphère produit (par réduction nitrogénasique) de l'ammonium qui conduira à la synthèse des composés azotés organiques (acides aminés). Elle fait intervenir des communautés bactériennes symbiotiques, spécifiques des végétaux auxquels ces bactéries sont associées (rhizobium, actinomycètes), des communautés bactériennes hétérotrophes aérobies ou anaérobies non symbiotiques ou dites «libres » et des communautés photolithotrophes (par exemple, cyanobactéries).

- La minéralisation de "l'azote organique » implique, dans une première étape qui conduit de l'azote organique à l'ammonium, l'ensemble ou une très grande partie des communautés bactériennes hétérotrophes aérobies et anaérobies et fongiques. Dans une seconde étape, la production de nitrate (oxydation de $\mathrm{NH}_{4}$ en $\mathrm{NO}^{-}{ }_{3}$ ) n'implique que des bactéries très spécialisées et concerne pour une grande part des populations bactériennes autotrophes (chimiolithotrophes) aérobies.

- L'immobilisation de l'azote, en fait l'absorption de $\mathrm{NO}_{3}$ ou $\mathrm{NH}_{4}$ pour des besoins nutritionnels, concerne pratiquement l'ensemble des microorganismes dans diverses conditions de milieux aérobies ou anaérobies.

- La dénitrification (dite aussi " dénitrification dissimilative 》) est une respiration anaérobie qui fait intervenir des populations bactériennes, surtout hétérotrophes (chimio-organotrophes), mais aussi autotrophes (chimiolithotrophes) (par exemple, Thiobacillus denitrificans) qui, en conditions anoxiques, assurent la production d'oxydes d'azote et en particulier $\mathrm{N}_{2} \mathrm{O}$, d'azote moléculaire $\mathrm{N}_{2}$, mais aussi $\mathrm{NH}_{3}$.

Récemment, on a observé que des organismes non définis, présents dans des eaux usées ou des boues d'épuration, peuvent, en conditions anaérobies, oxyder $\mathrm{NH}_{4}{ }^{+}$en azote moléculaire. Cette réaction désignée par anamox lanoxic ammonia oxidation) est exergonique et n'est pas associée à des activités métaboliques des bactéries nitrifiantes en conditions anaérobies. Toujours est-il qu'elle démontre, contrairement à ce qui semblait bien établi (!) que l'ammonium n'est pas stable en conditions anaérobies et qu'il peut être oxydé par des bactéries autres que les bactéries nitrifiantes (Madigan et al., 2000). 
On constate que chaque grande étape du cycle de l'azote présente des spécificités de communautés et populations microbiennes et de conditions de milieux plus ou moins particulières et que des couplages avec le cycle du carbone se manifestent pour :

- I'ammonification (minéralisation des matières organiques);

- la nitrification (fixation de $\mathrm{CO}_{2}$ en autotrophie);

- la fixation d'azote;

- la dénitrification hétérotrophe associées à la biodégradation et minéralisation de composés organiques source de carbone et d'énergie.

Comme on le constate pour d'autres types de processus microbiens et d'autres cycles (cycle du soufre ou du fer), un même processus peut avoir pour la production végétale, la qualité des sols, des eaux, de l'air..., des effets ou applications bénéfiques ou utiles ou au contraire des effets délétères ou nuisibles. Ainsi, la nitrification, essentielle au fonctionnement du cycle de l'azote et à la production d'azote assimilable pour les végétaux et les micro-organismes est aussi impliquée dans l'acidification des eaux, la contamination des nappes, la dissolution d'aluminium sous formes ioniques toxiques pour la faune aquatique, les végétaux... La dénitrification, autre processus essentiel du cycle de l'azote, étape fondamentale du retour de l'azote moléculaire $N_{2}$ vers l'atmosphère, processus d'épuration d'eaux contaminées par les nitrates, est aussi à l'origine des pertes de fertilisants azotés et de production de gaz à effet de serre $\left(\mathrm{N}_{2} \mathrm{O}\right)$.

Si ce cycle de l'azote paraît aussi relativement bien connu, il n'en reste pas moins d'un point de vue fondamental que les communautés microbiennes et en particulier bactériennes impliquées, leur dynamique, les paramètres qui contrôlent leur activité (disponibilité des sources de carbone et d'énergie, état hydrique du sol. . .) sont encore à préciser, voire à déterminer. Les applications et impacts concernent la production de gaz à effet de serre, les pratiques culturales et la production végétale, la qualité ef le traitement des eaux. Le développement des connaissances fondamentales et des applications nécessite aussi l'utilisation de modèles.

\subsection{Cycle du soufre}

Les transformations du soufre sont plus complexes que celles de l'azote en raison du plus grand nombre d'états d'oxydation. Trois formes paraissent dominantes dans les milieux naturels : sulfhydril, R-SH et sulfure $\mathrm{HS}^{-}(-2)$; soufre 
élémentaire, $\mathrm{S}_{0}(0)$; sulfate $\left(\mathrm{SO}_{4}{ }^{2-}\right)(+6)$. Certaines transformations comme l'oxydation des sulfures en conditions aérobies et $\mathrm{pH}$ neutre, impliquent tout autant les réactions chimiques que biologiques. Mais on distingue des grandes étapes où l'intervention des micro-organismes est fondamentale.

Pratiquement, l'ensemble des communautés microbiennes sont impliquées dans les processus de minéralisation aérobie ef anaérobie qui conduisent essentiellement aux sulfates et sulfures.

En conditions aérobies, acides ou neutres, des bactéries chimiolithotrophes oxydent, comme source d'énergie, les sulfures, le soufre élémentaire, le thiosulfate, le sulfite (Sulfolobus, Thiobacillus...) en utilisant $\mathrm{O}_{2}$ comme accepteur d'électrons pour assurer la fixation autotrophe du $\mathrm{CO}_{2}$. Quelques espèces (Th. denitrificans) peuvent aussi croître en anaérobiose en utilisant les nitrates comme accepteur d'électrons.

En conditions anaérobies strictes, les bactéries sulfatoréductrices utilisent les sulfates comme accepteur d'électrons (respiration anaérobie) pour le réduire en sulfure $\left(\mathrm{H}_{2} \mathrm{~S}\right)$. On désigne aussi ce processus par le terme " réduction dissimilative des sulfates". Elle contribue au fractionnement isotopique très significatif du soufre, l'isotope léger étant préférentiellement utilisé. Les donneurs d'électrons peuvent être pour ces bactéries sulfatoréductrices des composés organiques variés, mais aussi l'hydrogène (comme pour Desulfovibrio desulfuricans). On distingue par ailleurs une sulfatoréduction microbienne assimilative qui, par réduction des sulfates en $\mathrm{H}_{2} \mathrm{~S}$, conduit à la production de composés organiques soufrés (par exemple, cystéine, méthionine, ...) que mettent en œuvre de très nombreux micro-organismes pour leur nutrition et non pas pour leur respiration.

Ces deux grands types de processus, sulfo-oxydation et sulfatoréduction (dissimilative) bactériennes, ont des incidences et applications majeures et multiples (Ehrlich, 2002 ; Edwards ef al., 2001...). Outre l'implication directe sur le cycle du soufre, la sulfo-oxydation bactérienne intervient par l'oxydation-dissolution des sulfures sur l'acidification des eaux, des sols, la solubilisation et lixiviation (extraction par voie soluble) des métaux, la corrosion des bâtiments, ... La sulfatoréduction participe aux dépôts de sulfures métalliques et la formation de gisements, et est appliquée à l'épuration des eaux contaminées pour éliminer les métaux. Elle peut entraîner des effets toxiques $\left(\mathrm{H}_{2} \mathrm{~S}\right)$ vis-à-vis des végétaux...

Les différents processus microbiens du cycle du soufre assurent des couplages biologiques entre cycle du carbone, du soufre et du fer et même de l'azote (Th. denitrificans) et des couplages chimiques avec les réactions de changement d'état " solubilisation-dépôt » de nombreux métaux (cuivre, plomb, nickel, uranium, ...). On note aussi des analogies de conditions physicochimiques et biochimiques entre certaines étapes du cycle du soufre et de l'azote (par exemple, 
sulfo-oxydation et nitrification qui dépendent de bactéries autotrophes et aérobies; dénitrification et sulfatoréduction qui font intervenir des bactéries hétérotrophes et anaérobies).

\subsection{Cycle du phosphore}

Les transformations du phosphore font intervenir deux grands types de processus microbiens. L'un relativement spécifique met en œuvre des systèmes enzymatiques phosphatases qui hydrolysent les liaisons esters organiques phosphorés pour produire des phosphates minéraux assimilables par les micro-organismes et les végétaux. Dans les sols et les sédiments, tant le pool "phosphates organiques » que les diverses phosphatases et micro-organismes impliqués sont très mal connus (Golterman, 2004). L'autre principal type de processus fait intervenir, tant dans la rhizosphère que hors rhizosphère, des bactéries et champignons produisant des acides minéraux et organiques plus ou moins complexants. Ces acides excrétés dans le milieu, par échange entre protons des acides avec les cations des phosphates minéraux insolubles, libèrent des anions phosphates minéraux assimilables. C'est en faił un phénomène général d'altération microbienne des minéraux (Berthelin, 1988).

Outre l'assimilation du phosphore pour la croissance, certaines bactéries qualifiées de PAO (Phosphorus Accumulating Organism) peuvent stocker dans leur compartiment cytoplasmique le phosphore sous forme de granules de polyphosphates, au-delà de leur strict besoin métabolique. Cette propriété est utilisée en épuration des eaux pour favoriser un traitement biologique du phosphore plutôt qu'un traitement par précipitation chimique, qui entraîne une augmentation du volume des boues à traiter.

Cette suraccumulation du phosphore dans la cellule est régie par un métabolisme spécifique :

1. en condition anaérobie l'assimilation des acides gras volatils (issus de la fermentation de la matière organique) permet la synthèse de polyhydroxyalcanoates (PHA) en utilisant l'énergie libérée par l'hydrolyse des polyphosphates ;

2. en condition aérobie, l'hydrolyse des PHA fournit de l'énergie permettant une régénération des granules de polyphosphates, supérieure à l'hydrolyse observée en phase anaérobie.

Ce modèle métabolique reste à ce jour à confirmer (Mino, Van Loosdrecht et al., 1998). 
Les espèces impliquées ne sont que partiellement identifiées ( $\beta$ protéobactéries - Rhodocyclus spp. (Hesselmann, Werlen et al., 1999). À noter également que certaines bactéries appelées PAO dénitrifiantes (DPAO) peuvent utiliser le nitrate comme acceptateur d'électrons.

\subsection{Cycle du fer}

La mobilité du fer déterminée par le $\mathrm{pH}$, le Eh et la présence de ligands organiques, est sous la dépendance des activités microbiennes qui modifient et contrôlent en permanence ces paramètres (Ehrlich, 2002). En milieu acide ou neutre, aérobie ou micro-aérophile, des bactéries autotrophes (chimiolithotrophes) ou mixotrophes oxydent $\mathrm{Fe}^{l l}$ en $\mathrm{Fe}^{\text {III }}$ pour obtenir l'énergie nécessaire à leur croissance. Elles utilisent pour la plupart le $\mathrm{CO}_{2}$ comme source de carbone, certaines peuvent aussi oxyder les formes réduites du soufre et d'autres éléments $(U, A s, \ldots)$. Ces processus conduisent, sauf en conditions très acides ou complexantes, à la précipitation d'oxydes de fer sensu lato, de sulfates ou de phosphates (Ehrlich, 2002; Houot et Berthelin, 1992). Ces bactéries trouvent des applications dans la lixiviation ef l'extraction des métaux de minerais sulfurés, de matériaux pollués ou le traitement des eaux pour éliminer le fer, le manganèse et divers métaux qui peuvent $s^{\prime} y$ associer. $D^{\prime}$ autres communautés bactériennes aéro-anaérobies ou anaérobies, la plupart du temps hétérotrophes (chimio-organotrophes) utilisent Fe"ll comme accepteur d'électron pour leur respiration, en parallèle ou en complément de fermentations. Elles réduisent le fer en solution, mais entraînent aussi la dissolution et l'altération des oxydes ef oxyhydroxydes. Le fer est alors mobilisé et disponible sous forme de $\mathrm{Fe}^{\| l}$ et ne restera soluble qu'en conditions réductrices (Stemmler et Berthelin, 2003). Ce phénomène se produit en sol saturé, mais aussi quand la consommation d'oxygène entraîne l'anoxie. Ces bactéries se manifestent dans de nombreux sols sous conditions climatiques variées (tropicales humides, tempérées, montagnardes...). De tels phénomènes d'altération bactériennes des oxydes ferriques entraînent aussi une redistribution des métaux associés à ces oxydes vers des phases plus disponibles (hydrosolubles, échangeables, ...) (Quantin et al., 2002).

Par ailleurs, des champignons (mycorhizogène ou saprophytes) et des bactéries, rhizosphériques ou non, produisent des substances complexantes du fer (acides aliphatiques carboxyliques, acides phénols, acides hydroxamiques, ...) qui contribuent à l'altération de minéraux (ferromagnésiens, oxydes. . .) donc à la solubilisation d'éléments minéraux qui sont alors transférés plus abondamment aux solutions et aux végétaux (Leyval et Berthelin, 1991). Certains de ces composés, les sidérophores présentent une capacité complexante spécifique de $\mathrm{Fe}^{\text {III }}$ (Kraemer, 2004). 
Ces processus sont donc susceptibles de contrôler les dissolutions et néoformations de minéraux et modifient la disponibilité du fer et d'éléments associés dans les sols. Ils peuvent avoir des effets bénéfiques ou nocifs, directs ou indirects dans le fonctionnement des systèmes sols-plantes et se manifestent dans de nombreux couplages de cycles (avec carbone, soufre, phosphore, ou d'éléments en traces comme uranium, sélénium, arsenic. ..). La connaissance des structures et fonctions des communautés microbiennes impliquées et des paramètres du milieu qui les contrôlent ainsi que les couplages entre cycles est encore bien insuffisante.

\section{6 Éléments en traces}

Des éléments en traces comme le mercure, le chrome, le molybdène, le vanadium, l'uranium, le sélénium, le tellurium, ... peuvent subir divers types de transformations microbiennes : oxydation pour $\mathrm{Hg}, \mathrm{Cr}, \mathrm{Mo}, \mathrm{U}, \mathrm{Se}$; réduction pour $\mathrm{Hg}, \mathrm{Cr}, \mathrm{Mo}, \mathrm{V}, \mathrm{U}, \mathrm{Se}, \mathrm{Te}$; méthylation pour $\mathrm{Hg}$, Se, Te. Le cas du mercure à titre exemplaire peut être brièvement présenté (cf. aussi encadré chapitre 6.2). Dans l'atmosphère, le mercure est essentiellement présent sous la forme élémentaire $\left(\mathrm{Hg}^{\circ}\right)$ volatile, mais qui est oxydé photochimiquement en ion mercurique $\left(\mathrm{Hg}^{2+}\right)$ qui constitue la forme majeure d'introduction dans les sols et les eaux. Le mercure peut être facilement oxydé ou réduit $\left(\mathrm{Hg}^{\circ} \Leftrightarrow \mathrm{Hg}^{+}\right)$par des microorganismes du sol ou des sédiments soit directement soit par implication des bactéries sulfatoréductrices $\left(\mathrm{H}_{2} \mathrm{~S}+\mathrm{Hg}^{2+} \rightarrow \mathrm{HgS}\right)$ ou méthanogènes $\left(\mathrm{CH}_{3} \mathrm{Hg}^{+} \rightarrow\right.$ $\mathrm{CH}_{4}+\mathrm{Hg}^{\circ}$ ). Une réaction majeure, la méthylation qui fait intervenir une méthylcobalamine, conduit à une forme volatile environ 100 fois plus toxique que le mercure élémentaire $\left(\mathrm{Hg}^{\circ}\right)$ (Madigan et al., 2000 ; Ehrhich, 2002).

\section{Rôle des micro-organismes dans l'immobilisation et la solubilisation des métaux et non-métaux; application aux radionucléides (cf. aussi chapitre 4)}

Divers processus microbiens ou contrôlés par des activités microbiennes interviennent pour immobiliser et solubiliser les éléments majeurs, les éléments en traces ou encore les radionucléides. Ils sont impliqués notamment dans les phénomènes d'altération des minéraux et de transfert des éléments vers les plantes et les organismes. 


\subsection{Immobilisation des éléments minéraux (dont les radionuclèides) ${ }^{\prime}$}

L'immobilisation microbienne de radionucléides fait intervenir des processus de biosorption, bioaccumulation, réduction et précipitation qui se manifestent pour de nombreux éléments qu'ils soient ou non radioactifs.

\subsubsection{Biosorption}

La biosorption est généralement définie comme un processus non actif, c'està-dire ne nécessitant pas de consommation d'énergie par le micro-organisme. $C^{\prime}$ est en fait une sorption par des mécanismes physicochimiques (échange ionique, sorption, complexation). Elle implique différents niveaux de structure des cellules microbiennes: les parois et les membranes cellulaires externes et leurs composants, les organites intracellulaires.

Des constituants des parois fongiques et membranes bactériennes (polysaccharides, protéines, ...) possèdent des groupements fonctionnels $\left(-\mathrm{NH}_{2}\right.$, $-\mathrm{COOH},-\mathrm{OH},-\mathrm{PO}_{4} \mathrm{H}_{2}$ l qui ont des affinités pour les métaux, dont les radionucléides. Ils se comportent comme des polyanions naturels, sites potentiels de fixation des métaux et des radionucléides chargés positivement tels $\mathrm{Cd}^{2+}$, $\mathrm{Cs}^{+}, \mathrm{La}^{3+}, \mathrm{Eu}^{3+}$ et $\mathrm{Yb}^{3+}$ (Texier et al., 2000; Deneux-Mustin et al., 2003) (cf. tableau 7.11 .

\subsubsection{Bioaccumulation}

Ce sont des processus actifs démontrés chez de nombreux micro-organismes procaryotes et eucaryotes. Ils utilisent habituellement des systèmes de transport spécifiques et non spécifiques localisés à la surface de leurs membranes. Par exemple, le césium, tant radioactif que non radioactif, est accumulé par des micro-organismes utilisant les systèmes transmembranaires de transport du potassium (Bossemeyer et al., 1989) et parfois (surtout chez les cyanobactéries) en utilisant les systèmes transmembranaires de transport de l'ammonium (Avery et al., 1992).

Comme d'autres métaux, les radionucléides s'accumulent aussi dans les vacuoles de micro-organismes eucaryotes tels que les micro-algues et les champignons (Gadd, 1996). La fixation peut se faire sur des macromolécules libres ou appartenant à des structures intracellulaires.

\footnotetext{
${ }^{1}$ Remarque : la section 3 de ce chapitre concernant les micro-organismes et les radionucléides a été rédigée avec la participation de Christian Tamponnet, IRSN, DEI, SECRE, Cadarache.
} 


\subsubsection{Réduction}

Des réductions de radionucléides se produisent sans être liées au métabolisme énergétique et peuvent correspondre à des mécanismes de tolérance et de détoxification, comme c'est le cas du mercure (Madigan et al., 2000).

\subsubsection{Précipitation}

Après réduction, des éléments minéraux, radionucléides ou non, peuvent précipiter sous forme d'oxydes (pour le technétium), de sulfures (chez les bactéries sulfatoréductrices), ou de phosphates (bactéries phosphatase positives comme celles du genre citrobacter) précipitant les actinides tétravalents tels que Th(IV) et Pu(IV) (Tolley et al., 1995). Le neptunium(V) peut être éliminé par l'action combinée de Shewanella putrefaciens bioréduisant le neptunium(V) en neptunium(IV) et de citrobacter sp. le précipitant sous forme de phosphate (Lloyd et al., 2000).

\subsection{Solubilisation d'éléments minéraux dont les radionucléides par les micro- organismes}

De nombreux processus microbiens peuvent conduire à un accroissement de la mobilité des radionucléides. Dans les milieux terrestres, la solubilisation des radionucléides a des implications importantes dans leurs transferts aux eaux, aux plantes et aux autres organismes supérieurs. Cette solubilisation, souvent désignée par lixiviation, fait intervenir des processus d'oxydation ou de production de métabolites acides et complexants (Berthelin, 1988 ; Deneux-Mustin et al., 2003...).

Ainsi en condition aérobie, les bactéries autotrophes comme Thiobacillus ferrooxidans interviennent par oxydation des sulfures avec production d'oxydant (sulfate ferrique) et d'acide sulfurique pour extraire l'uranium qui est solubilisé sous forme de sulfate d'uranyle.

Par ailleurs, des bactéries et des champignons peuvent produire des protons, des acides organiques et des agents chélateurs, tels que les acides carboxyliques, les acides phénols et les sidérophores qui solubilisent les métaux dont les radionucléides et accroissent leur mobilité dans le sol (Munier-Lamy et Berthelin, 1987).

Certaines réactions de réduction entraînent directement ou indirectement une solubilisation de radionucléides. Par exemple, le plutonium est solubilisé par des 
bactéries réductrices du fer et le radium et l'uranium peuvent être solubilisés par des bactéries réductrices des sulfates et du fer (Landa et Gray, 1995).

La dégradation microbienne de la matière organique fixant des métaux peut conduire à leur solubilisation.

\subsection{Rôle spécifique des champignons mycorhiziens}

Les associations symbiotiques mycorhiziennes (racines-champignons) interviennent selon les conditions expérimentales (plantes, champignons, éléments, conditions de culture) soit pour limiter, soit pour améliorer le transfert de radionucléides aux plantes (Deneux-Mustin et al., 2003). Ce sont des phénomènes et mécanismes qu'il y a lieu de préciser.

\subsection{Tableau d'ensemble}

Le tableau 7.1 présente à titre d'illustration un résumé des processus microbiens qui, soit directement (réduction, oxydation, méthylation, bio-accumulationbiosorption), soit indirectement (en agissant sur les phases porteuses de métal ou en modifiant les conditions de milieu : dissolution des oxydes, dissolution ou formation de sulfures, ... l, interviennent dans les changements d'état de quelques éléments en traces et donc dans le cycle de ces éléments ( $d$ 'après Deneux-Mustin et al., 2003).

L'importance des processus signalés ou connus est indiquée par + (peu important) à ++++ (processus majeur).

\section{4 | Les couplages de cycles}

\subsection{Couplages entre processus biotiques}

Les couplages du cycle du carbone avec les autres cycles (azote, soufre, phosphore, fer, ... évoqués ci-dessus, sont connus ou relativement bien connus d'un point de vue des mécanismes microbiens impliqués, mais sont loin d'être définis du point de vue de l'écologie fonctionnelle. Les paramètres biotiques (nature, dynamique, activité des populations impliquées) et abiotiques (paramètres physiques et chimiques du milieu) de régulation sont à mieux définir. 


\begin{tabular}{|c|c|c|c|c|c|c|c|c|c|c|c|c|c|}
\hline Elements & $\mathrm{m}$ & $A s$ & $\mathrm{Cd}$ & $\mathrm{Cs}$ & $\mathrm{Cr}$ & Mi: & $\mathrm{bb}$ & Pu & $R a$ & 5 & $\mathrm{Tc}$ & Th & $U$ \\
\hline Reduction & & ++++ & & & ++ & & & + & & +++ & + & & ++ \\
\hline Oxydation & & ++++ & & & & & & & & ++ & & & + \\
\hline Acidification dons la thizosphere & +++ & ++ & +++ & + & ++ & +++ & $+t$ & ++ & ++ & + & + & + & + \\
\hline Production de composés chelctants & ++ & + & ++ & & + & ++ & ++ & + & + & + & + & ++ & +++ \\
\hline Biodegradation MO & ++ & + & ++ & + & + & ++ & ++ & ++ & + & + & + & & + \\
\hline Formotion hydroxides Fe thr & & & & & & & & & & ++ & & & ++ \\
\hline Biosorption-bioaccumulation & ++ & & +++ & ++ & ++ & ++ & ++ & +++ & + & & + & + & + \\
\hline Formation du 5 insoluble & & ++ & + & & & + & & & & ++ & + & & \\
\hline Role des mycomizes & & + & + & ++ & + & + & + & & & + & & & \\
\hline Méthylation & & ++ & & & & & & & & & & & \\
\hline Reduction Fe, Mn s & & +++ & & & & ++ & & & ++ & + & + & & ++ \\
\hline
\end{tabular}

Tableau 7.1

Exemples de processus microbiens, qui interviennent directement ou indirectement (voir textel, dans la mobilité et le transfert de quelques éléments en traces choisis à titre d'exemple (d'après Deneux-Mustin et al., 2003). 
Les couplages les plus simples concernant majoritairement deux cycles biogéochimiques correspondent essentiellement :

- à des phases de minéralisation des matières organiques impliquant carbone, phosphore, azote, soufre..., où la nature des composés organiques, leur disponibilité et biodégradabilité, les paramètres de régulation sont à préciser : par exemple, comment se développe et est contrôlée la minéralisation de l'azote ou du phosphore sous divers peuplements forestiers?

- à des processus d'oxydation de substrats minéraux et de fixation de $\mathrm{CO}_{2}$ et qui concernent la nitrification, la sulfo-oxydation, l'oxydation du fer par des bactéries autotrophes;

- à des processus de réduction comme la dénitrification, la réduction du fer, la sulfatoréduction dont la mise en œuvre et la régulation sont à bien déterminer.

Ces couplages biologiques peuvent concerner plusieurs cycles comme par exemple, $\mathrm{C}, \mathrm{S}$ et $\mathrm{Fe}$ au cours de l'oxydation-altération-dissolution des mineraux sulfurés par Thiobacillus ferrooxidans ou C, S, N pour la bactérie Thiobacillus denitrificans avec des implications fortes dans le fonctionnement des milieux naturels ou dans des applications au traitement de minerais ou à la pollution et au traitement des eaux.

En fait, il est absolument nécessaire de considérer les diverses étapes des cycles biogéochimiques, non seulement par élément mais aussi par association de cycles ou d'étapes de cycles, pour établir une approche fonctionnelle.

\subsection{Couplages entre processus biotiques et abiotiques}

Pour indiquer l'incidence de ces couplages, la figure 7.2 souligne les associations qui peuvent se manifester entre le fer, l'uranium et le soufre, sans cependant faire apparaître les relations avec le cycle du carbone qui est étroitement associé à ceux du fer et du soufre. Des essais de modélisation sont encourageants et à développer (Dassonville et al., 2004).

La bactérie Thiobacillus ferrooxidans, en oxydant le fer et le soufre des sulfures (pyrite), va produire un oxydant sous forme de sulfate ferrique qui va pouvoir oxyder l'uranium $\mathrm{UO}_{2}(\mathrm{U} \mathrm{IV})$ en $\mathrm{UO}_{2}{ }^{2+}(\mathrm{U} \mathrm{VI})$ qui deviendra soluble, sous forme de sulfate, en milieu acide. L'acide sulfurique est produit par l'oxydation du soufre élémentaire ou des sulfures (pyrite) par T. ferroxidans ou par 


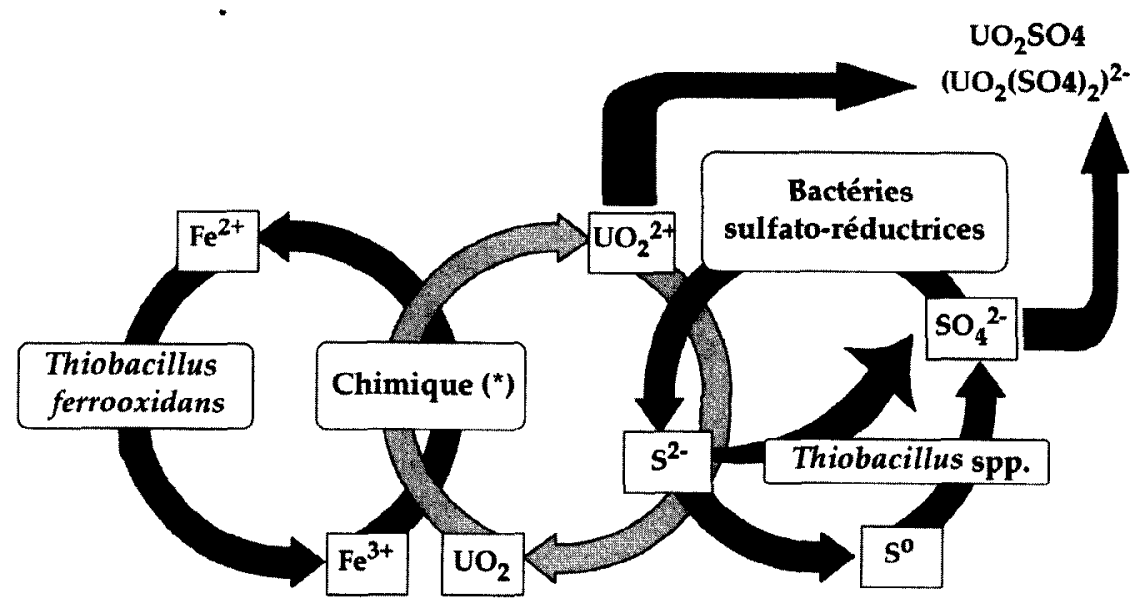

(*) : peut être bactérien

Figure 7.2

Exemple de couplage entre cycles du fer, de l'uramium et du soufre lla réduction du fer est majoritairement bactérienne).

des bactéries de même type fonctionnel. L'uranium U(VI) soluble pourra être réduit en présence de bactéries sulfatoréductrices formant des sulfures $\left(\mathrm{S}^{2-}\right)$. Le fer réduit et les sulfures pourront être réoxydés par $T$. ferrooxidans ou des bactéries de même type fonctionnel. Enfin le fer III peut être réduit par des bactéries ferriréductrices.

Le carbone est aussi associé à ces boucles d'oxydo-réduction en apportant le $\mathrm{CO}_{2}$ qui sera fixé en autotrophie par exemple par $T$. ferrooxidans et les matières organiques qui seront oxydées, soit par les bactéries sulfatoréductrices, soit par les bactéries ferriréductrices. Les différentes phases concernant le fer et le soufre sont essentiellement biotiques et la boucle concernant l'uranium est essentiellement abiotique.

\section{5 | Rôle de la rhizosphère}

La rhizosphère, définie pour la première fois voilà un siècle, correspond aux parties des sols sous l'influence des racines des plantes, des micro-organismes, symbiotiques et non symbiotiques, et de la faune qui leur sont associés. Les racines exercent des effets mécaniques, prélèvent des nutriments, produisent et libèrent des composés organiques et minéraux solides, liquides, gazeux. Le sol fournit l'eau, les nutriments, le support solide organisé. Les micro-organismes et 
la faune du sol au voisinage des racines y trouvent des conditions énergétiques et nutritionnelles favorables à leur croissance et activité. Dans de telles conditions de milieu, les organismes vivants " racines-micro-organismes-faune » interagiront entre eux et avec les constituants organiques et minéraux des sols. La rhizosphère est ainsi une niche écologique qui « éveille » et stimule les diverses activités microbiennes qui participeront très significativement au fonctionnement du cycle de nutriments majeurs et des oligo-éléments carbone, azote, phosphore, sélénium, fer, ... et aux transformations qui conduiront à des transferts vers les plantes, les eaux, l'atmosphère. La rhizosphère est ainsi le théâtre de processus de transformation de minéraux (par exemple, altération, ...) intervenant dans le cycle des éléments associés au cycle de l'eau et dont les acteurs et leurs jeux sont encore bien mal connus (Gobat et al., 2003).

\section{Les associations faune - micro-organismes}

Malgré le rôle majeur, maintenant bien reconnu, de la faune du sol dans les transferts de matière et d'énergie par brassage et les impacts physiques et chimiques qui peuvent en résulter, peu de travaux concernent les interactions faune/micro-organismes. Pourtant des effets bénéfiques de telles associations ont été rapportés voici déjà quelques dizaines d'années (Dommergues et Mangenot, 1970) en constatant que l'association bactéries-collemboles favorisait par exemple la cellulolyse par une action mécanique de fractionnement des matériaux et par dispersion des bactéries cellulolytiques. Il paraît important de mieux définir ces interactions et le rôle qu'elles peuvent jouer dans la dynamique du cycle du carbone, de l'azote, du phosphore et du soufre (Lavelle et Spain, 2001 ; Gobat et al., 2003).

\section{Applications aux biotechnologies de l'environnement et aux traitements de matériaux, minerais et déchets}

L'ensemble des mécanismes microbiens qui interviennent dans le fonctionnement des cycles biogéochimiques trouve des applications dans différents domaines des biotechnologies appliquées au traitement :

1. des pollutions organiques et minérales des sols, des eaux et de l'air;

2. des résidus et déchets; 
3. des matières minérales et des minerais (extraction, accumulation de métaux et non métaux) (Berthelin, 1987; Ehrlich et Brierley, 1990) (cf. aussi les chapitres 6.1 et 6.2 de ce rapport).

Ces applications concernent aussi certains aspects de la production et de la qualité des végétaux.

Ainsi, pour le cycle du carbone et de l'azote, les mécanismes microbiens actuellement mis en œuvre ou en perspectives d'application concernent par exemple la biodégradation de polluants organiques ou la production (biosynthèse) de composés d'intérêt industriels ou médicaux..., l'élimination des nitrates des eaux (dénitrification), la fixation d'azote moléculaire pour la production végétale. . Une "ingénierie environnementale » et le développement de biotechnologies appliquées au contrôle de la production de gaz à effet de serre paraissent prometteurs.

D'autres cycles (fer, soufre, ...) sont aussi très concernés par l'extraction de métaux des minerais (Ehrlich 2002; Edwards et al., 2001), lors de leur élimination des eaux ou de résidus ou minéraux industriels. Des applications apparaissent pour de nombreux éléments majeurs et en traces, afin de les éliminer d'effluents en les accumulant par des phénomènes de biosorption, bioaccumulation...

Il y a lieu de souligner que la connaissance des mécanismes microbiens intervenant dans le fonctionnement des cycles biogéochimiques et la définition des paramètres qui les contrôlent peuvent conduire à des applications dont le champ s'élargit en contribuant au développement de ce que l'on peut qualifier de biotechnologies de l'environnement, de biotechnologies minérales (par exemple, biohydrométallurgie pour l'extraction et accumulation de métaux).

Bien souvent ces développements se font ou se feront en associant des méthodes biologiques aux méthodes physiques et chimiques.

\section{Des outils prometteurs pour le développement de l'écologie microbienne des milieux naturels}

Les méthodes de culture des micro-organismes sur des milieux nutritifs ne permettent d'isoler et cultiver qu'une faible partie (de l'ordre de $1 \%$ ) des microorganismes des milieux naturels (sols, sédiments, océans...). 
Des méthodes récentes de biologie moléculaire ont été appliquées depuis 15 à 20 ans aux micro-organismes de ces milieux et en particulier des sols. Elles permettent d'obtenir des profils moléculaires des populations et communautés microbiennes, de caractériser des micro-organismes, de découvrir des bactéries non cultivables à partir d'extraction, purification, séquençage d'ADN et amplification de séquences d'ADN (Prosser, 2002; Kirk et al., 2004).

Pour passer de ces méthodes, qui contribuent à mettre en évidence des potentiels génétiques, à des mises en évidence d'activités, l'amplification d'ARN ribosomal et de gènes de fonctions fournit déjà et pourra fournir des informations tant sur la dynamique que sur les activités in situ des populations bactériennes. L'utilisation des techniques moléculaires a déjà contribué à des progrès significatifs sur la connaissance de divers mécanismes (fixation d'azote, nitrification, dénitrification, biodégradation de polluants organiques).

À ces méthodes "génomiques " appliquées aux ADN et ARN s'ajoute l'étude des protéines (protéomique) qui complète la recherche et caractérisation d'activités (recherche d'enzymes). Enfin des outils chimiques et physiques (marquage aux isotopes stables ${ }^{13} \mathrm{C},{ }^{15} \mathrm{~N}$... de molécules substrat et par voie de conséquence des acides nucléiques; microscopie confocale avec marquage par des sondes plus ou moins spécifiques fluorescentes...) permettent d'une part des mesures encore plus spécifiques d'activités, et d'autre part d'observation in situ plus précises et en 3D.

Un numéro récent de Biofutur $\left(n^{\circ} 268,2006\right)$ présente plusieurs articles qui soulignent le développement et l'intérêt qu'apportent la génomique, la protéomique, le traçage moléculaire et isotopique au développement de l'écologie microbienne du sol.

Toutefois, les approches moléculaires d'écologie microbienne du sol ne constituent pas une nouvelle science mais sont des outils qui ouvrent, comme le soulignent Gobat et al. (2003), de nouveaux horizons dans la connaissance des relations entre processus, paramètres les contrôlant, dynamique et activités des populations ou communautés impliquées.

\section{Conclusions et perspectives}

Les micro-organismes (bactéries et champignons) interviennent très significativement dans le fonctionnement des cycles biogéochimiques soit seuls, soit en association avec les plantes et la faune. Ils se manifestent dans toutes les enveloppes superficielles de la planète : sols, sédiments, eaux douces, océans. 
De nombreux mécanismes (oxydation, réduction, production de substances complexantes, méthylation, biosorption, bioaccumulation. . .) sont déjà relativement bien connus, tant d'un point de vue physiologique, biochimique, génétique qu'écologique, mais des lacunes dans les connaissances d'intérêt fondamental et appliqué persistent.

Elles doivent être comblées aussi bien pour la compréhension que pour la gestion de phénomènes allant des changements climatiques (production de $\mathrm{CO}_{2}$, $\mathrm{N}_{2} \mathrm{O}, \mathrm{CH}_{4} \ldots$. ) à l'extraction des métaux (biohydrométallurgie), en passant par la gestion des écosystèmes lato sensu (par exemple : pratiques forestières, pratiques agricoles...), le traitement des pollutions, la gestion des résidus et déchets organiques et/ou minéraux...

Seulement quelques pour cent (\%) (voire pas plus de $1 \%$ ) des microorganismes sont cultivables et connus. Les méthodes de biologie moléculaire (Prosser, 2002; Kirk et al., 2004, Biofutur, 2006), qui ont considérablement progressé ces dix à vingt dernières années, ont contribué bien sûr à la connaissance des organismes, à la mise en évidence d'organismes non cultivables ef à une meilleure connaissance de certaines étapes de cycles biogéochimiques. Mais la seule étude de la diversité et de la dynamique de populations est encore insuffisante. La connaissance des micro-organismes ne peut pas se limiter à des études de populations, mais a besoin de se développer en intégrant la nature, la dynamique et l'activité (fonctions) des communautés et populations. Le développement de la génomique et de la protéomique associées à des outils chimiques et physiques va dans ce sens (Biofutur, 2006). II y a lieu de faire progresser une connaissance fonctionnelle prenant en compte l'ensemble des facteurs biologiques, chimiques et physiques des milieux ainsi que les couplages de cycles.

Tout d'abord plusieurs domaines, qui vont de la physiologie, la biochimie et la génétique des organismes à l'écologie fonctionnelle des populations et communautés, ont à progresser, et il ne faut pas se limiter à quelques organismes dont la représentativité n'est absolument pas établie (par exemple : Shewanella putrefaciens ou Geobacter sp. pour la réduction du fer).

La définition des relations entre nature, dynamique et activité des communautés et populations implique un plus grand investissement dans la connaissance des processus et des paramètres aux interfaces (micro-organismes-minéraux, par exemple).

Les valeurs seuils des paramètres qui orientent et déterminent les activités microbiennes, disponibilité des substrats et des nutriments, propriétés physiques, physicochimiques des milieux létat hydrique, conditions redox et acidobasiques...), sont à vraiment prendre en compte. Les sites d'activités et de 
" conservation 》 des organismes (par exemple : rhizosphère, litières et horizons humifères, agrégats, drilosphère, termitosphère...) ont à être mieux introduits dans une démarche fonctionnelle; de même que les interactions entre organismes " micro-organismes/racines/faune " pour lesquelles de grosses lacunes demeurent.

Une interdisciplinarité obligatoire doit conduire à l'intégration des paramètres biotiques et abiotiques à différentes échelles d'espace et de temps pour l'acquisition de connaissances fondamentales, le développement de modèles et d'une ingénierie écologique scientifique et technique ou à des biotechnologies de qualité. En fait, il faut veiller au développement d'une écologie microbienne prenant en compte les paramètres abiotiques (physiques et chimiques) déterminants des milieux.

Enfin, l'étude de la biosphère souterraine profonde ouvre un champ de recherche particulièrement vaste pour l'acquisition de nouvelles connaissances concernant la vie dans les milieux extrêmes et dont les attendus, tant fondamentaux qu'appliqués, sont très prometteurs. 


\title{
Note sur la biosphère souterraine profonde
}

\author{
ALAIN-YVES HUC, FRANCK HAESELER, BERNARD OLLVIER, MICHEL MAGOT \\ ET DANIEL PRIEUR
}

Ce n'est que très récemment que l'existence d'une biosphère souterraine profonde a été largement acceptée par la communauté scientifique. Le scepticisme initial était fondé sur des préoccupations quant à la provenance des organismes détectés dans les échantillons ramenés à la surface. Les progrès réalisés dans la qualité des prélèvements et des méthodes de caractérisation moléculaire des micro-organismes ont permis de lever cette hypothèque.

Les difficultés d'obtention d'échantillons représentatifs et le coût des forages ont conduit à utiliser comme sites de prélèvements les gisements pétroliers, mines, sites expérimentaux de stockage profond de déchets nucléaires mais aussi ceux de grands programmes de recherche internationaux, comme IODP (International Ocean Drilling Program).

La mise en évidence de ces populations bactériennes profondes pose les questions de la limite ultime de cette vie et des formes de vies extrêmes actives dans ces environnements. Au cours des dix dernières années, les forages scientifiques profonds, notamment ceux des campagnes ODP (Ocean Drilling Program) ont commencé à explorer cette biosphère largement inconnue. Les premiers résultats indiquent que des écosystèmes bactériens sont présents dans la croûte océanique et dans des sédiments marins jusqu'à des profondeurs d'enfouissement jusqu'ici considérées comme incompatibles avec la vie (cf. figures 7.3 et 7.4). Les écosystèmes subterrestres profonds, aujourd'hui reconnus jusqu'à plusieurs centaines de mètres, se caractérisent par l'absence $d^{\prime}$ 'oxygène (anaérobiose) et la pauvreté en nutriments, en particulier azote et phosphore et des cinétiques d'activités faibles. 


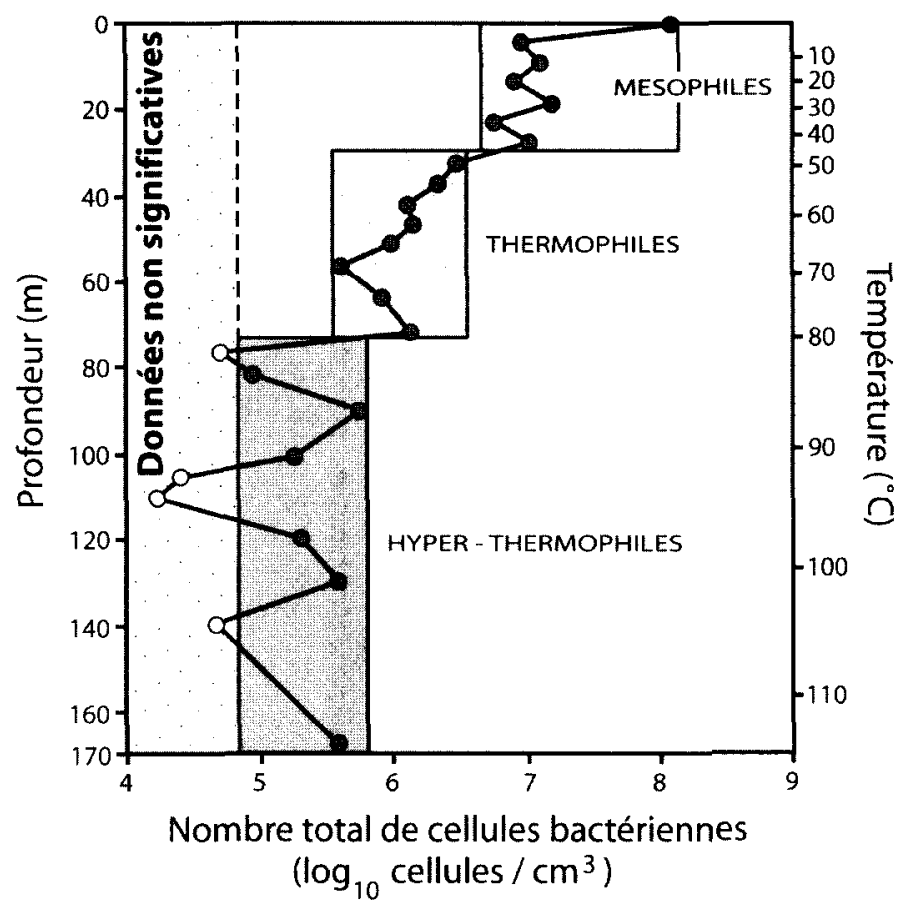

Figure 7.3

Distribution des bactéries en fonction de la profondeur ef de la température, dans le site hydrothermal de San Juan de Fuca (Leg 169) (ingebritsen et al, 2000).

Ces écosystèmes hébergent une biodiversité dont la majorité des représentants sont inconnus dans d'autres écosystèmes.

La température est un facteur limitant majeur pour l'établissement de communautés bactériennes. On estime une température limite maximale théorique comprise entre $120^{\circ} \mathrm{C}$ et $150^{\circ} \mathrm{C}$. Notons que des travaux récents, concernant une bactérie nommée "bactérie 121 », ont vérifié la possibilité de vie bactérienne à $121^{\circ} \mathrm{C}$.

Certains sédiments profondément enfouis contiennent encore des populations de l'ordre de $10^{5}$ à $10^{6}$ cellules par $\mathrm{cm}^{3}$. Ces chiffres peuvent croître dans des environnements disposant de sources de carbone et d'énergie (par exemple, méthane ou présence simultanée de $\mathrm{CO}_{2}$ et de $\mathrm{H}_{2}$ ). Les observations disponibles (incluant des indices de biodégradation effective de champs de pétrole) semblent indiquer que la biosphère crustale profonde peut atteindre 3 à $4 \mathrm{~km}$.

Le nombre de cellules par unité de volume est relativement modeste, cependant l'énorme quantité de sédiments (estimée à $510^{25} \mathrm{~cm}^{3}$ ) pourrait accueillir 


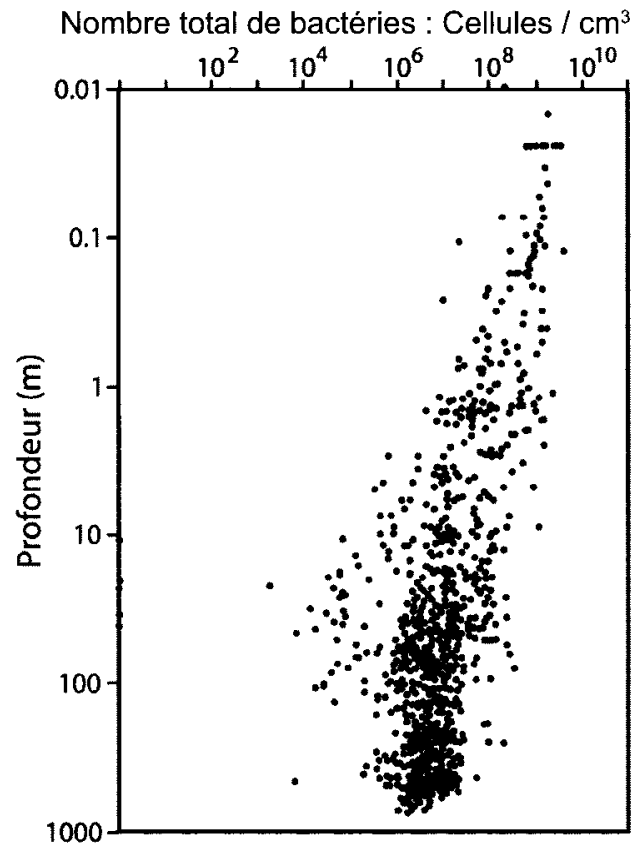

Figure 7.4

Distribution du nombre total de bactéries en fonction de la profondeur. Données provenant de 17 sites du programme "Ocean Drilling Project 》 (Cragg et al., 1997; Parkes et al., 2000).

un nombre total de cellules bactériennes de l'ordre de $10^{30}$. Bien que ce chiffre soit à prendre avec précaution, en raison des incertitudes sur les mesures et du faible nombre de points d'échantillonnage ainsi extrapolés, une telle valeur représenterait $90 \%$ de la biomasse bactérienne sur Terre. Cette biomasse bactérienne profonde pourrait contenir $3-510^{17} \mathrm{~g}$ de carbone, ce qui représenterait 60 à $100 \%$ du carbone présent dans la biomasse globale.

Ces micro-organismes profonds pourraient avoir un rôle non négligeable en tant que réservoir dans les cycles globaux du carbone, du soufre, de l'azote et du phosphore.

L'activité de cette biosphère microbienne enfouie pourrait également jover un rôle significatif sur la composition de l'atmosphère et sur les climats, mais aussi dans des phénomènes chimiques, généralement considérés comme abiotiques dans ces domaines.

La biosphère profonde semble ainsi être le siège d'activités chimioautotrophes avec utilisation du $\mathrm{CO}_{2}$ / carbonates comme source de carbone et de l'hydrogène comme source d'énergie. Dans ce contexte, les archaebactéries 
méthanogènes jouent un rôle primordial in situ, mais aussi certaines bactéries sulfatoréductrices et les bactéries acétogènes. Avec l'enfouissement, et l'augmentation concomitante de température, certains composés inorganiques sont susceptibles d'être activés, tels que des minéraux contenant des métaux (fer, ...) fournissant de nouveaux accepteurs d'électrons en profondeur. De plus, ces minéraux sont à même de produire de l'hydrogène via l'oxydation du $\mathrm{Fe}^{++}$couplée à la réduction de l'eau. Les conditions régnant à grande profondeur peuvent donc permettre des réactions abiotiques, favorisant l'activité bactérienne, différentes de celles se produisant à la surface de la Terre.

L'impact de cette biosphère profonde sur les grands cycles biogéochimiques est à ce jour pratiquement inconnu. Enfin le type de métabolisme à l'œuvre sera à prendre en considération dans les recherches menées dans le cadre du stockage souterrain du $\mathrm{CO}_{2}$ pour lutter contre les gaz à effet de serre.

\section{Références bibliographiques}

Avery SV., Codd GA. et Gadd GM. (1992). Caesium transport in the cyanobacterium Anabaena variabilis : kinetics and evidence for uptake via ammonium transport system(s). FEMS Microbiol Lett, 95 : 253-258.

Bada JL. (2004). How life began on earth : a status report. Earth and Planetary Science Letters, $226: 1-15$.

Berthelin J. (1987). Des bactéries pour extraire des métaux. La Recherche, 188 : 720-725.

Berthelin J. (1988). Microbial weathering processes in natural environments, In Lerman A. and Meybeck M. (eds). Physical and chemical weathering in geochemical cycles. Kluwer Academic Press, London, 33-59.

Biofutur (2006). Écologie microbienne des sols, 268 : 21-57.

Bossemeyer D., Schlösser A. et Bakker EP. (1989). Specific cesium transport via the Escherichia coli Kup (TrkD) K+ uptake system. J Bacteriol, 171 : 2219 . 2221.

Cragg BA., Law KM., Cramp A. et Parkes RJ. (1998). The response of bacterial populations to sapropels in deep sediments of the eastern mediterranean (site 969) : AHF. In Robertson KC., Emeis C., Richter et Camerlenghi A. (eds). Proceedings of the Ocean Drilling. Program, Scientific Results, 160 : 303-307.

Dassonville F., Renault P. et Vallés V. (2004). A model to describe the interactions between anaerobic microbiology and geochemixstry in a soil amended with glucose and nitrate. Eur J Soil Sci, 55 : 29-45. 
Deneux-Mustin S., Roussel-Debet S., Mustin C., Henner P., Munier-Lamy C., Colle C., Berthelin J., Garnier-Laplace J. et Leyval C. (2003). Mobilité et transfert racinaire des éléments en trace : influence des micro-organismes du sol. Éditions Tec \& Doc, Paris.

Dommergues Y. et Mangenot F. (1970). Écologie microbienne du sol. Masson, Paris.

Edwards KJ., Hu B., Hamers JR. et Banfield JF. (2001). A new look at microbial leaching patterns on sulfide minerals. FEMS Microbial Ecology, 34 : 197206.

Ehrlich HL. et Brierley CL. (1990). Microbial Mineral Recovery. McGraw-Hill Publ Co, New York.

Ehrlich HL. (2002). Geomicrobiology. 4th edition Marcel Dekker Inc., New York.

Gadd GM. (1996). Role of microorganisms in the environmental fate of radionuclides. Endeavour, 20 : 150-156.

Ghiorse WC. (1997). Subterranean life. Science, 275 : 789.

Gobat JM., Aragno M. et Matthey W. (2003). Le sol vivant. Presses polytechniques et universitaires romandes. Lausanne. $2^{\mathrm{e}}$ édition.

Gold T. (1998). The Deep Hot Biosphere. Copernicus and Springer-Verlag, $225 \mathrm{p}$.

Golterman HL. (2004). The chemistry of phosphate and nitrogen compounds in sediments. Kluwer Academic Publishers.

Head IM., Jones DM. et Larter SR. (2003). Biological activity in the deep subsurface and the origin of heavy oil. Nature, $426: 344-352$.

Hesselmann RPX., Werlen C. et al. (1999). Enrichment, phylogenetic analysis and detection of a bacterium that performs enhenced biological phosphate removal in activated sludge. System Appl Microbiol, 22 : 454-165.

Houot S. et Berthelin J. (1992). Submicroscopic studies of iron deposits occuring in field drains : formation and evolution. Geoderma, 52 : 209-222.

Ingebritsen SE., Sanford NE. et Toth J. (2000). Recent studies on bacterial populations and processes in subseafloor sediments. Hydrology Journal, 8 : 11-28.

Jeanthon C., Reysenbach AL., L'Haridon AL., Gambacorta S., Pace A., Glénat NR. et Prieur D. (1995). Hermotoga subterranea sp. nov., a new thermophilic bacterium isolated from a continental oil reservoir. Arch Microbiol, 164 : 91 97. 
Kirk JL., Beaudette LA., Hart M., Moutoglis P., Klironomos JN., Lee H. et Trevors JT. (2004). Methods of studying soil microbial diversity. Journal of Microbiological Methods, 58 : 169-188.

Kormas KA., Smith D., Edgcomb V. ef Teske A. (2003). Molecular analysis of deep subsurface microbial communities in Nankai trough sediments IODP Leg 190, Site 1176). FEMS Microbiology Ecology, 45 : 115-125.

Kraemer SM. (2004). Iron oxide dissolution and solubility in the presence of siderophores. Aquat Sci, 66 : 3-18.

Landa ER. et Gray JR. (1995). US Geological survey : research on the environmental fate of uranium mining and milling wastes. Environ Geol, 26 : 19-31.

Lavelle P. et Spain AV. (2001). Soil ecology. Kluwer Academic Publishers, Dordrecht.

Leyval C. et Berthelin J. (1991). Weathering of a mica by roots and rhizospheric microorganisms of Pine. Soil Sci Soc Am J, 55 : 1009-1016.

Lloyd JR., Yong P. et Macaskie LE. (2000). Biological reduction and removal of $\mathrm{Np}(\mathrm{V})$ by two microorganisms. Environ Sci Technol, 34 (7) : 1297-1301.

Madigan MT., Martinko JM. et Parker J. (2000). Brock Biology of microorganisms. Prentice Hall International, Upper Saddle River, (9th edition).

Magot M., Ollivier B. et Patel KC. (2000). Microbiology of Petroleum Reservoirs. Antonie van Leeuwenhoek, 77 : 1003-116.

Mino T. et van Loosdrecht MCM. (1998). Microbiology and biochemistry of the enhanced biological phosphate removal process. Wat Res, 32 (11) : 31933207.

Munier-Lamy C. et Berthelin J. (1987). Formation of polyelectrolyte complexes with major (Fe, Al) and traces (U, Cu) elements during heterotrophic microbial leaching of rocks. Geomicrobiol J, 5 : 119-147.

Parkes RJ., Cragg BA. et Wellbury P. (2000). Recent studies on bacterial populations and processes in subseafloor sediments. Hydrogeol Journ, 8 : 11-28.

Pedersen K. (2000). Exploration of Deep intraterrestrial microbial life : current perspectives. FEMS Microbiology Letters, 185 : 9-16.

Prosser J. (2002). Molecular and functional diversity in soil microorganisms. Plant and Soil, 244 : 9-17.

Quantin C., Becquer Th., Rouiller J. et Berthelin J. (2002). Redistribution of metals in a New Caledonia Ferrasol after microbial weathering. Soil Sci Am J, 66 : 1797-1804. 
Stemmler S., Berthelin J. (2003). Microbial activity as a major factor in the mobilization of iron in the humid tropic. Eur J Soil Sci, 54 : 725-733.

Stevens TO. et McKinley JP. (1995). Lithoautotrophic microbial ecosystems in deep basalt aquifers. Science, $270: 270-271$.

Texier AC., Andrès Y., Illemassene M. et Le Cloirec P. (2000). Characterisation of Lanthanide ions binding sites in the cell wall of Pseudomonas aeruginosa. Environ Sci Technol, 34 : 610-615.

Tolley MR., Strachan LF. et Macaskie LM. (1995). Lanthanum accumulation from acidic solutions using a Citrobacter sp. Immobilized in a flow-through bioreactor. J Industrial Microbiol, $14:$ 271-280.

Torsvik V., Goksoyr J. et Daae FL. (1990). High diversity in DNA of soil bacteria. Appl Environ Microbiol, 56 : 782-787.

van Elsas JD., Frois-Duarte G., Keijzer-Wolters A. et Smit E. (2000). Analysis of the dynamics of fungal communities in soil via fungal PCR of soilDNA followed by denaturing gradient gel electrophoresis. J Microbiol Methods, 43 : 133-151.

Wellsbury P., Mather I. et Parkes RJ. (2002). Geomicrobiology of deep, low organic carbon sediments in the woodlark Basin, Pacific Ocean. FEMS Microbiology Ecology, 42 : 59-70. 
CHAPITRE 8

\section{Nature, rôle et fonctions des matières organiques dans les sols ${ }^{1}$}

JACQUES BERTHELIN 



\section{Les matières organiques du sol et leurs grandes fonctions}

Comparativement au petit volume qu'ils occupent à la surface des continents, les sols contiennent de grandes quantités de matières organiques «mortes" (distribuées de façon variable entre résidus végétaux, animaux ef microbiens, acides humiques $(\mathrm{AH})$, acides fulviques (AF), humine), dont la masse selon divers auteurs est de l'ordre de 1,5 à 3,0 10 12 t pour l'ensemble des continents. Mis à part les litières (horizon $\mathrm{OL}$ ), les matières organiques des sols sont associées plus ou moins étroitement ou en mélange avec les constituants minéraux. La plus grande partie est insoluble dans l'eau, et moins de $1 \%$ se trouve sous forme dissoute ou particulaire dans les eaux superficielles. Le temps de résidence moyen des matières organiques dans les sols, estimé selon certains auteurs à 30-40 ans ou 15 ans pour d'autres (Balesdent et al., 2005) souligne un renouvellement rapide et montre bien le rôle des organismes et en particulier des micro-organismes dans leur biotransformation, biodégradation et minéralisation. Toutefois ce temps de résidence court inclut celui des matières organiques fraîches (compris entre 0,5 et 5 ans, Paul et Huang, 1980) et ne doit pas masquer la stabilité des substances humiques sensu lato avec des âges plus ou moins longs : par exemple, des fractions organiques d'un horizon d'accumulation d'argiles (horizon BT) d'un sol brun lessivé (Luvisol) atteignent 4800 ans.

Les matières organiques du sol, qui proviennent de la décomposition et de la transformation des matériaux végétaux par des processus biotiques el abiotiques, ne constituent pas seulement un compartiment majeur du cycle terrestre du carbone ( $c f$. chapitre 1), mais sont aussi impliquées fondamentalement dans d'autres processus et fonctions d'un grand intérêt (Andreux, 1996; Andreux et Munier-Lamy, 1994 ; Berthelin et al., 1999 ; Balesdent et al., 2005) :

- les cycles des éléments majeurs et en traces et de nutriments organiques et minéraux (carbone, azote, phosphore, soufre, fer, potassium, calcium, magnésium. . I pour les micro-organismes, les plantes, les animaux;

- les sources et les stocks d'énergie (produits organiques et minéraux, donneurs et accepteurs d'électrons) pour les micro-organismes;

- la réactivité chimique et physicochimique des sols par leurs implications dans les phénomènes d'échange, de complexation, de réduction, d'oxydation...;

- la rétention de métaux toxiques et de produits organiques contaminants (Lamy et al., 2005; Berthelin et al., 2005);

- les propriétés physiques comme l'organisation et la structure des sols, leurs caractéristiques hydrauliques, ... I'aération et la réserve utile en eau... 
Ce sont ces aspects, autres que ceux impliqués dans le cycle général du carbone et la production de gaz à effet de serre, qui seront présentés dans ce chapitre pour souligner l'intérêt d'une meilleure connaissance de divers types de propriétés ef fonctions des matières organiques des sols.

\section{2 | Nature des matières organiques}

Les matières organiques des sols les «plus stables " mais aussi chimiquement les plus "actives » sont constituées par les substances humiques sensu lato dont la localisation et la distribution dans les différents compartiments des sols peuvent être déterminées par des fractionnements physiques (agrégats et/ou particules de diverses tailles : argiles, limons, sables) et à la fois chimiques et physiques (extraction, précipitation par des réactifs alcalins ou acides, encombrement moléculaire. .. . S'agissant le plus souvent de macromolécules, on peut aussi les caractériser morphologiquement (méthodes microscopiques, submicroscopiques et ultramicroscopiques associées éventuellement à des observations et mesures spectroscopiques : par exemple, microscope électronique à balayage, microscope électronique à transmission couplé à un spectromètre à perte d'énergie d'électrons...).

Leur caractérisation chimique, physicochimique, physique et biologique (biodégradabilité) montre que les matières organiques, en fonction de leurs matériaux d'origine et des conditions biotiques et abiotiques qui contrôlent leur transformation et leur évolution, constituent pour l'ensemble des sols un " continuum " de composés allant des AF (acides fulviques) (les plus simples et les plus solubles dans une large gamme de $\mathrm{pH}$ ), aux $\mathrm{AH}$ (acides humiques) (solubles uniquement en milieu alcalin) et à l'humine (les plus stables et non dispersables à tout $\mathrm{pH}$ ). On les distinguera d'abord par leur milieu d'origine, puis par la méthode utilisée pour les isoler, et finalement par leurs caractéristiques analytiques. Parmi ces dernières, on retiendra particulièrement leur composition élémentaire (carbone, hydrogène, azote, oxygène), leurs propriétés macromoléculaires (encombrements moléculaires et polydispersité) et colloïdales de dispersion et coagulation (variations des propriétés de surface selon la concentration dans l'eau, la salinité ou le $\mathrm{pH}$ ), leur stabilité chimique et physique (hydrolyse acide ou alcaline, stabilité thermique), leur aliphaticité et aromaticité. On insistera aussi sur leurs principaux groupements fonctionnels $\left(-\mathrm{COOH},-\mathrm{OH},-\mathrm{OCH}_{3},-\mathrm{C}=\mathrm{O}\right.$, $-\mathrm{NH}_{2},-\mathrm{SH},-\mathrm{PO}_{4} \mathrm{H}_{2} \ldots$ ) et leur biodégradabilité et capacité à stocker et fournir des sources d'énergie et des nutriments (Andreux et Munier-Lamy, 1994; Berthelin et al., 1999).

Les teneurs en carbone $(\mathrm{C})$ et en azote $(\mathrm{N})$ des acides fulviques et des acides humiques, la part du carbone et de l'azote du sol qu'ils représentent et leurs proportions relatives (rapport $\mathrm{AF} / \mathrm{AH}$ ), ont longtemps constitué le principal critère 
de caractérisation de la matière organique des horizons et des sols. Les teneurs élémentaires les plus courantes sont incluses dans les gammes suivantes : $40 \%<$ $\mathrm{C}<55 \% ; 30 \%<\mathrm{O}<50 \% ; 3,5 \%<\mathrm{H}<8 \% ; 0 \%<\mathrm{N}<6 \%$. Le soufre et le phosphore organiques ne dépassent en général pas $1 \%$ mais représentent une source importante de ces éléments dont la nature est par ailleurs mal définie. Les AF présentent en général les teneurs les plus basses en carbone, mais les teneurs les plus élevées en oxygène et en hydrogène. Les rapports atomiques oxygène/carbone sont donc proches de 1,0 dans certains acides fulviques et de 0,5 dans les acides humiques les plus évolués. Les rapports atomiques hydrogène/carbone, qui traduisent l'indice d'aromaticité, dépassent 1 dans la plupart des acides fulviques et les acides humiques les moins aromatiques et peuvent atteindre 1,5 dans les acides humiques les plus aromatiques. Les résultats des méthodes dégradatives (pyrolyse-spectrométrie de masse) ou spectroscopiques (absorption infrarouge, RMN du proton et $d u^{13} \mathrm{C}$ ) se corrèlent avec les précédents et ont permis d'importantes avancées concernant la structure chimique des matières humiques (Hayes et al., 1989; Kögel-Knabner, 2000). Des méthodes comme la spectrométrie de masse à résonance cyclotronique ionique à transformée de Fourier (FT-ICR-MS) apparaissent très prometteuses pour caractériser des mélanges complexes de matières organiques en permettant de définir les grands types de familles chimiques (par exemple, lipides, protéines, cellulose, lignine, hydrocarbures polyaromatiques. . .) et de suivre leur évolution en les replaçant dans un diagramme de van Krevelen) (Kujawinski, 2002; Kujawinski et al., 2004). Les méthodes utilisant le marquage isotopique ${ }^{15} \mathrm{~N}$ et ${ }^{13} \mathrm{C}$ déjà utilisées sont d'un grand intérêt et doivent se développer, en relation avec l'écologie microbienne, pour étudier la dynamique de ces matières organiques (cf. chapitre 7).

Inversement, les teneurs en azote sont très variables selon les milieux et les méthodes d'extraction, mais sont plus élevées dans les acides humiques que les acides fulviques (Andreux et Munier-Lamy, 1994). Pour certains auteurs (Schnitzer, 1985), la présence d'azote dans les acides fulviques serait même un artefact d'extraction, en raison du faible degré d'incorporation des biomolécules (protéines, aminosucres et bases organiques azotées). En revanche, dans les acides humiques, une part prépondérante de l'azote se trouve incorporée sous forme de liaisons covalentes entre les groupements $-\mathrm{NH}_{2}(\mathrm{NH})$ des biomolécules ef les cycles aromatiques ou quinoniques issus des composés polyphénoliques (des tanins, de la lignine ou de pigments végétaux ou animaux) les plus oxydables. Cet azote fait alors partie intégrante de la structure des acides humiques lou de certains acides fulviques), polycondensats de couleur brun-noir dont la masse moléculaire peut atteindre entre $10^{4}$ et $10^{6}$ daltons (dalton $=$ unité de masse atomique égale à la fraction $1 / 12$ de la masse du nucléide ${ }^{12} \mathrm{C}$ ). Entre 40 et $60 \%$ de cet azote résiste alors à toute séparation ou coupure hydrolytique et échappe à toute identification (Andreux et Munier-Lamy, 1994; Andreux, 1996). 


\section{3 | Réactivité chimique et physicochimique}

Les acides fulviques, composés les plus solubles, faiblement teintés en jaune ou en brun clair et de plus faible « masse » moléculaire (de quelques centaines à quelques milliers de daltons) possèdent en général la plus grande teneur en groupement fonctionnels $-\mathrm{COOH}$ et $-\mathrm{OH}$ et la plus forte réactivité chimique. La connaissance de ces groupements fonctionnels est d'un intérêt majeur, car ils déterminent les propriétés et comportements des acides fulviques et acides humiques et leur réactivité vis-à-vis des composés organiques et inorganiques, solubles et insolubles, constituants du sol ou polluants. Les groupes fonctionnels acides, carboxyliques et phénoliques $(-\mathrm{COOH}$ et $-\mathrm{OH})$ représentent plus de $50 \%$ des atomes d'oxygène des acides fulviques et acides humiques. Ces groupements fonctionnels constituent un ensemble de charges variables qui, des milieux acides aux milieux neutres ou légèrement alcalins, présentent des domaines allant des fortes aux faibles acidités. Les acides fulviques, dont les groupements acides restent dissociés à $\mathrm{pH}$ plus faible que ceux des acides humiques, possèdent une activité ou réactivité plus élevée que ces derniers, ce qui leur confère une capacité complexante des métaux plus forte. En effet, en se dissociant, en fonction du $\mathrm{pH}$ du milieu, ces groupements fonctionnels $(-\mathrm{COOH} \rightarrow$ $\mathrm{COO}^{-} \mathrm{H}^{+},-\mathrm{OH} \rightarrow-\mathrm{O}^{-} \mathrm{H}^{+}$) sont à l'origine de phénomènes de complexation d'éléments métalliques majeurs et en traces (McBride, 1994; Andreux et Munier-Lamy 1994 ; Berthelin et al., 1999 ; Lamy et al., 2005. . ). La figure 8.1 représente schématiquement l'état de substances humiques dans différents domaines de $\mathrm{pH}$. Pour les milieux d'acidité forte à très forte, les groupements carboxyliques et phénoliques ne sont pas dissociés et les composés humiques sont floculés-condensés. En milieu alcalin, ils seront dispersés et les groupements fonctionnels seront dissociés. Les parois des champignons et les membranes des bactéries possèdent aussi de tels groupements fonctionnels qui leur confèrent des propriétés de fixation de métaux.

Le tableau 8.1 présente des valeurs de l'équivalent d'acidité totale pour différents domaines d'acidité en distinguant les acidités fortes, faibles, très faibles pour un acide fulvique, un acide humique et les parois du mycélium de deux champignons. Dans ce tableau figurent aussi des valeurs moyennes de constantes de dissociation $(\mathrm{pKm})$ pour les domaines d'acidités dites « faibles » ef "très faibles". L'acidité relativement importante des acides fulviques et acides humiques est à relier à l'origine essentiellement microbienne de ces composés. Pour des acides humiques purifiés de chernozems, podzols, rendzines, les acidités totales vont de 260 à $460 \mathrm{c} \mathrm{mol} \mathrm{kg}^{-1}\left(1\right.$ centi mole kg $\left.{ }^{-1}=1 \mathrm{me} / 100 \mathrm{~g}\right)$.

Les matières organiques du sol réagissent aussi par des interactions organiques-organiques qui conduisent à la formation de "résidus liés 》 en fait de liaisons chimiques avec des composés organiques exogènes contaminants 


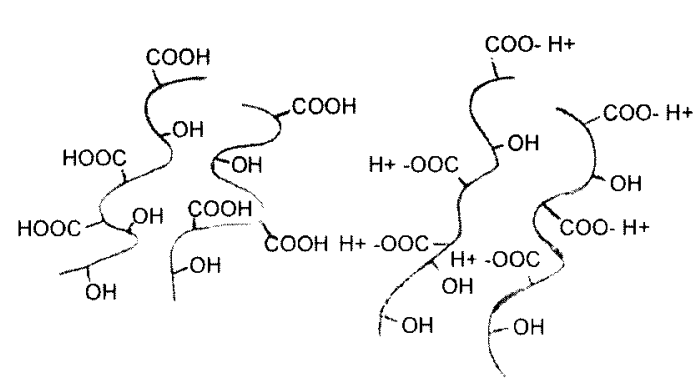

(I)

(II)

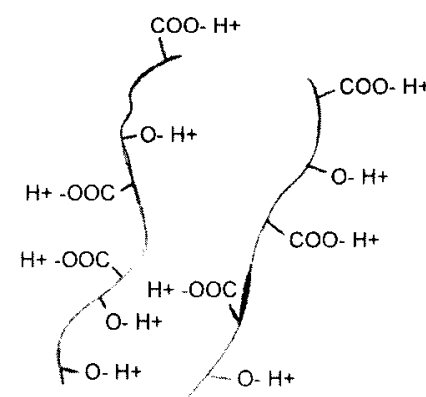

(III)

\section{Figure 8.1}

Représentation schématique très simplifiée de substances humiques à divers $\mathrm{pH}$ : (I) faible $\mathrm{pH}$ (milieu très ocide). (III) pH moyen (milieu peu acide ou neutre). (III) pH élevé (milieu alcalin) (d'après Mc Bride, 1994; Berthelin et al., 2005).

\begin{tabular}{|c|c|c|c|c|c|}
\hline & \multirow{2}{*}{ Acidité forte } & \multicolumn{2}{|c|}{ Acidité faible } & \multicolumn{2}{|c|}{ Acidiré très faible } \\
\hline & & c mol $\mathrm{kg}^{-1}$ & $\mathrm{pK}_{\mathrm{m}}$ & $\mathrm{cmol} \mathrm{kg}^{-1}$ & $p K_{m}$ \\
\hline $\mathrm{AF}$ & 170 & 292 & 4,8 & 94 & 10,1 \\
\hline $\mathrm{AH}$ & 72 & 421 & 5,7 & 140 & 10,4 \\
\hline A. niger & 15 & 19 & 7,0 & 23 & 10,4 \\
\hline R. arhizus & 48 & 38 & 6,5 & 138 & 9,2 \\
\hline
\end{tabular}

Tableau 8.1

Valeurs de différents domaines d'acidités (fortes, faibles, très faibles) (en $\mathrm{c} \mathrm{mol} \mathrm{kg}^{-1}$ ) pour un acide fulvique $(A F$ ), un acide humique (AH) purifiés et les parois de deux champignons (Aspergillus niger et Rhizopus arrhizus). Constantes de dissociation moyennes $\left(p K_{m}\right)$ des domaines d'acidité faible et très faible (d'après Andreux et Munier-Lamy, 1994 ; Berthelin et al., 2005).

(pesticides lato sensu, hydrocarbures aromatiques polycycliques HAP). Ces interactions sont sous la dépendance de divers types de liaisons chimiques actuellement mal définies (Andreux et Munier-Lamy, 1994; Berthelin et al., 1999; Benoît et al., 2000; Barriuso ef al., 2005). Toutefois, on peut distinguer des liaisons à haute énergie comme les liaisons ioniques et les liaisons covalentes ef des liaisons à plus faible énergie comme les liaisons hydrogène ou les liaisons de London-van der Waals. Les mécanismes impliqués sont étudiés essentiellement avec des modèles de polymères humiques (Andreux et Munier-Lamy 1994 ; Berthelin et al., 1999). 


\section{Encadrë 8.1}

\section{Les matières organiques solubles et particulaires}

Les matières organiques, sous forme dissoute ou particulaire, des solutions du sol ne constituent qu' une infime part des matières organiques totales du sol mais présentent un grand intérêt fonctionnel (Zsolnay, 2003; Dudal et Gérard, 2004). Elles forment la fraction la plus disponible chimiquement et biologiquement et contribuent aux phénomènes d'altération, de nutrition et à l'activité des micro-organismes (Marschner et Kalbitz, 2003), de transfert de métaux et de contaminants organiques (Amiri et al., 2005). Leur nature va dépendre bien sûr du type de sol, des conditions environnementales et des modes de prélèvements. En effet, les matières organiques des eaux à écoulement gravitaire présentent des teneurs et une nature qui seront différentes de celles des eaux dites " capillaires ". Leur étude se développent avec de nouvelles approches qui permettent de mieux les caractériser et de définir leurs fonctions (Benedetti et al, 2002 ; Zsolnay, 2003; Dudal et Gérard, 2004...)

\section{4 | Biodégradation et stabilisation des matières organiques}

Les matières organiques, qui constituent une source de carbone, d'énergie et de nutriments pour les organismes vivants du sol, faune et micro-organismes, sont biodégradées, biotransformées et biominéralisées par des processus biochimiques impliquant pour la plus grande part des micro-organismes (bactéries et champignons). Différentes cinétiques et différentes voies de biodégradation et biotransformation se manifestent et dépendent de paramètres comme les conditions climatiques (pluviométrie, température), la nature et la gestion des sols, la nature et la disponibilité des produits organiques, la présence, la survie et l'activité des micro-organismes (Dommergues et Mangenot, 1970; Berthelin et al., 1994 ; Gobat et al., 2003...). Globalement, 55 à $70 \%$ des résidus animaux et végétaux qui arrivent au sol retournent à l'atmosphère sous forme de $\mathrm{CO}_{2}$ l'année suivante, mais des variabilités importantes s'observent. Par exemple, il ne restera que $36 \%$ du carbone d'une paille de blé, une année après son apport, alors que l'on retrouvera $83 \%$ du carbone pour des mousses de tourbières. Les matières organiques résiduelles (biomasse microbienne, produits de métabolisme, produits transformés, produits hérités des matériaux végétaux. . .) s'associeront plus ou moins étroitement aux substances humiques et aux constituants minéraux des sols. Ces produits résiduels sont alors plus ou moins stabilisés 
dans des structures organominérales (de la structure moléculaire aux macroagrégats) (figures $8.2,8.3$ et 8.4 ) et seront soumis à de nouveaux cycles de biodégradation et minéralisation. Les associations de composés organiques aux matières organiques humifiées et/ou aux constituants minéraux du sol favorisent en général la stabilisation et limitent la biodégradation. C'est le cas pour divers composés organiques associés aux argiles et/ou stabilisés dans des agrégats de petites tailles (limons) ou de protéines ou polysaccharides incorporés dans des substances humiques ou bien encore fixés par sorption sur les constituants minéraux et organominéraux du sol.

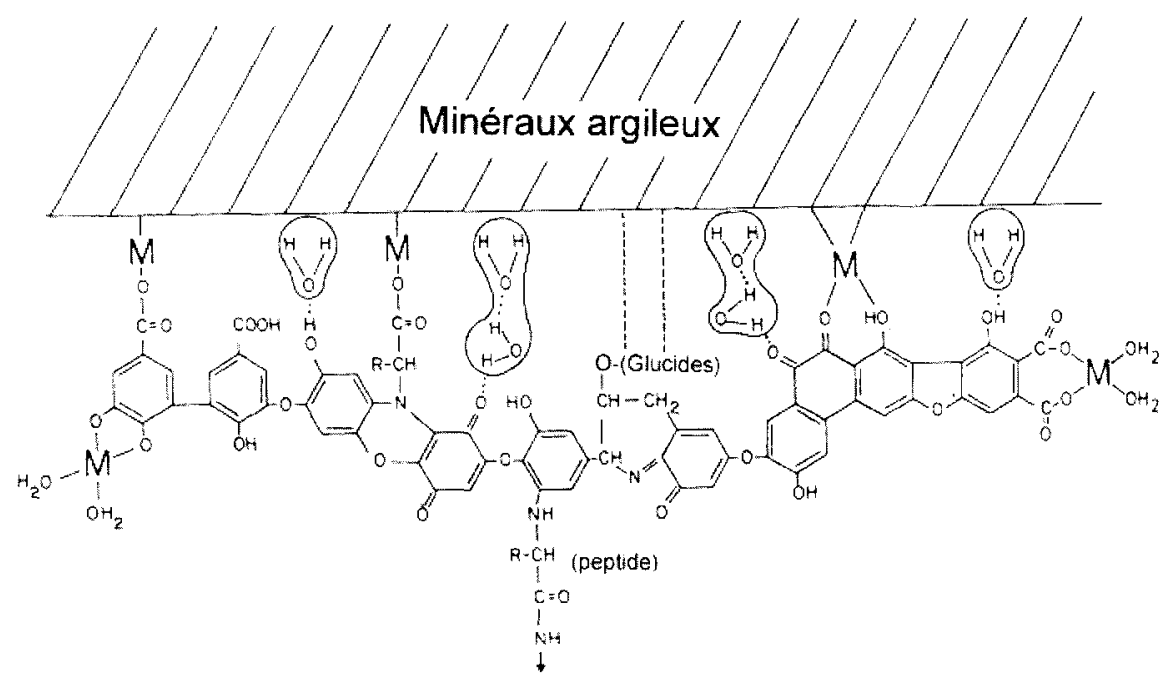

Figure 8.2

Modèle d'association organominérale (d'après Stevenson et Ardakani, in Berthelin et al., 1999).

Ces associations organominérales participent aux propriétés physiques /stabilité structurale, résistance à l'érosion et au compactage, aération, réserve en eau) des sols.

Les bactéries et les champignons chimio-organotrophes du sol peuvent, dans des conditions de milieu très variées, utiliser l'ensemble des composés organiques apportés ou présents dans le sol et même dégrader des produits chimiques autres que ceux fournis par le règne végétal et animal (substances xénobiotiques). Si les conditions de milieu sont favorables, les micro-organismes hétérotrophes transforment les matières organiques $d^{\prime}$ 'origine végétale, animale, microbienne, en nouveaux corps microbiens, en produits de métabolisme gazeux, liquides, solides qui sont finalement minéralisés $\left(\mathrm{CO}_{2}, \mathrm{H}, \mathrm{O}, \mathrm{NH}_{3}\right.$, $\left.\mathrm{NO}_{3}, \mathrm{SO}_{4}, \mathrm{PO}_{4}, \ldots\right)$. Une fraction des produits de métabolisme ou des constituants cellulaires sont relativement peu biodégradables ou acquièrent, dans les sols, une certaine stabilité en formant des macromolécules plus ou moins 


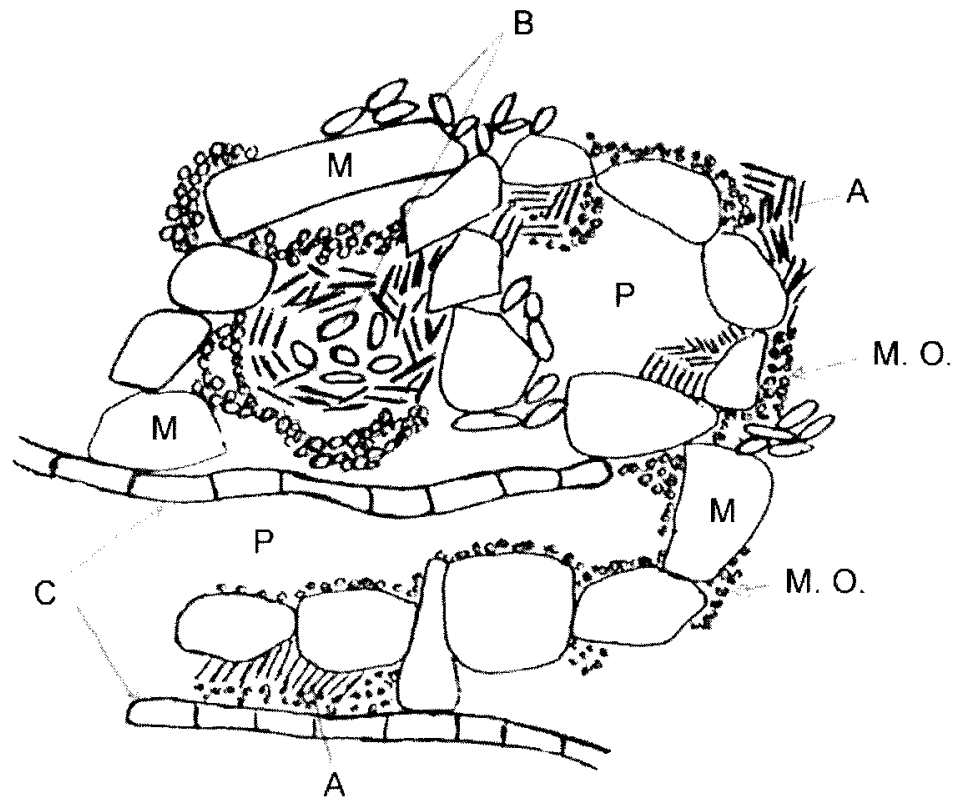

Figure 8.3

Schéma d'un agrégat organominéral: $M$ (minéraux); $B$ (bactéries); $C$ (mycélium de champignon); $A$ (argile), $M O$ (matière organique et oxyhydroxydes); $P$ (pores) (d'après Berthelin, 2005).

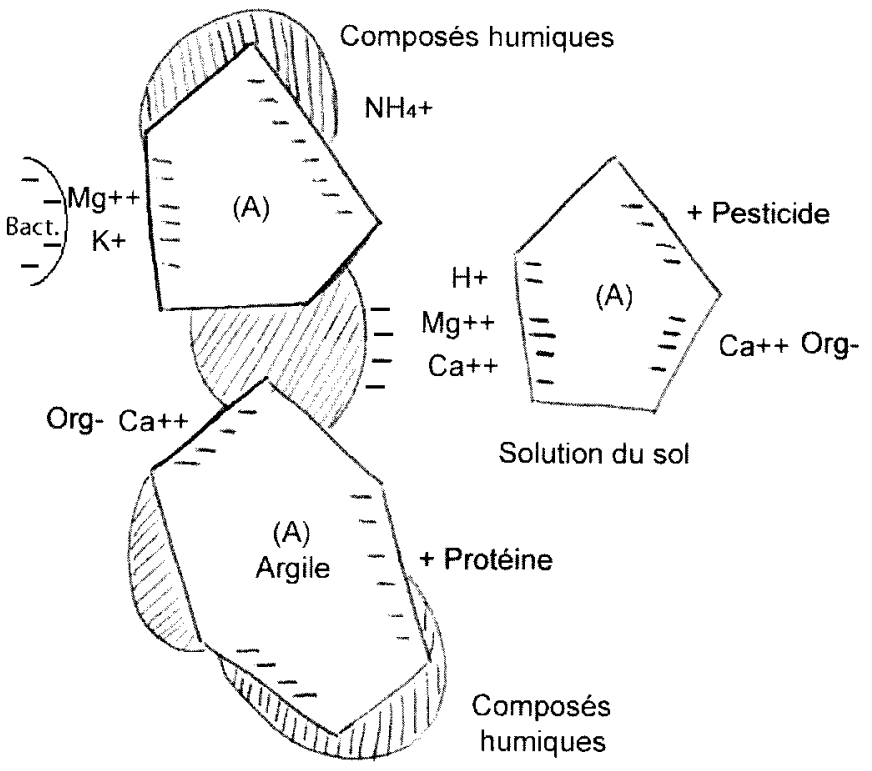

\section{Figure 8.4}

Représentation schématique d'interactions entre particules argileuses, argilohumiques, ions minéraux et organiques et bactéries (bact.) (d'après Berthelin, 2005). 
polycondensées ou bien en s'associant à des ions métalliques ou à des supports minéraux (Quiquampoix, 2000; Chenu, 2001) (figures 8.2, 8.3 et 8.4). Différents processus peuvent être à l'origine de cette transformation, qui est absolument nécessaire... On distingue encore mal la part des processus physicochimiques éventuellement catalysés par des enzymes dites "libres " de celle de la biodégradation proprement dite. Des échanges et transferts se produisent du compartiment des matières organiques stables (peu biodégradables) vers le compartiment des matières organiques "actives " plus facilement biodégradables. Ce compartiment « actif » doit être continuellement alimenté par des matières organiques fraîches pour présenter une activité microbienne "suffisante » nécessaire au maintien des fonctions et de la structure du sol. La dégradation, la transformation et l'évolution physicochimique des matières organiques (Adrian et al., 1986) devraient être mieux distinguées des phénomènes biologiques.

Par exemple, l'équipe de P. Albrecht (Hebting et al., 2006) a démontré que la formation du pétrole est principalement associée à des réactions chimiques, les réactions biologiques n'étant que préliminaires (voir encadré 8.2).

En général, les glucides, protéines et peptides sont facilement dégradables par de nombreux micro-organismes (la microflore dite " ammonifiante " qui assure la minéralisation des acides aminés en produisant de l'ammoniaque, donne une image voisine de celle de la communauté microbienne totale cultivable). La biodégradation des glucides et composés protéiques conduit non seulement à $\mathrm{CO}_{2}, \mathrm{H}_{2} \mathrm{O}, \mathrm{NH}_{3}, \mathrm{NO}_{3}$, mais aussi à d'autres polysaccharides, des peptides, des composés phénoliques, des composés aminés qui, par des processus d'oxydation biotique et abiotique, de polymérisation, de formation de complexes organominéraux, sont à la base de la formation des polymères humiques (Andreux et Munier-Lamy, 1994 ; Berthelin et al., 1994 ; Gobat et al., 2003). Un point particulier, propre au sol, concerne la biodégradation des substances phénoliques (lignine) qui fait intervenir des communautés spécialisées, les champignons de pourritures blanches, des pourritures molles et des pourritures brunes impliquées dans la décomposition des structures polyphénoliques avant que d'autres organismes comme par exemple des bactéries prennent le relais pour poursuivre l'oxydation et la minéralisation de ces substances. Les productions de substances humiques font directement intervenir des activités fongiques et bactériennes, impliquées dans la formation de polymères phénoliques, de pigments colorés, de composés préhumiques (phénols, acides phénols) très mal définies.

Les parois fongiques et membranes bactériennes possèdent des constituants (polysaccharides, protéines, lipopolysaccharides, ...) qui portent, tout comme les substances humiques, des groupements fonctionnels $\left(-\mathrm{NH}_{2},-\mathrm{COOH},-\mathrm{OH}\right.$, $-\mathrm{PO}_{4} \mathrm{H}_{2}, \ldots$ ) qui leur confèrent des propriétés d'échanges anioniques et cationiques et de complexation des métaux. Le tableau 8.1 présente des valeurs de différents domaines d'acidités fortes, faibles, très faibles, qui sont portées par 
les groupements fonctionnels des constituants des parois de deux champignons. Des acidités totales de surface de micro-organismes peuvent présenter des valeurs très élevées comme par exemple pour la bactérie Thiobacillus ferrooxidans (397 $\mathrm{c} \mathrm{mol} \mathrm{kg}{ }^{-1}$ ou 397 me pour $100 \mathrm{~g}$ de matière sèche) d'un point de vue physicochimique et, indépendamment de leurs fonctions biochimiques, les bactéries, en raison de leur petite taille 10,5 à $2 \mu \mathrm{m}$ pour la plus grande part) et de ces charges de surface variables, possèdent au même titre que les argiles, les oxyhydroxydes, les composés humiques, des propriétés colloïdales. Les propriétés de surface doivent les faire considérer comme des agents à forte réactivité physicochimique.

\section{5 | Interactions organominérales et structure des sols}

Le concept $d$ 'interactions entre les composés organiques et minéraux des sols est probablement aussi vieux que la science du sol. Toutefois, la nature des associations organominérales est relativement mal connue. Ce concept d'associations organominérales peut recouvrir tant les complexes organominéraux (Bruckert, 1994) que les complexes argilo-humiques (Chenu, 2001) qui sont tous deux des entités semblables et correspondent à des associations "polymères organiques (composés humiques ou polysaccharidiques...)-cationsargiles » et dont les structures de base sont présentées très simplement par les figures $8.2,8.3$ et 8.4 .

Cette définition doit en fait être élargie à l'ensemble des associations organominérales impliquant non seulement les argiles, mais aussi les oxydes, oxyhydroxydes et les carbonates qui sont des pièges efficaces pour les matières organiques et d'excellents ciments pour assurer la stabilité des agrégats. Vrdoljak et Sposito (2002) soulignent l'importance et l'intérêt d'une bonne définition de l'état de surface des minéraux et du type de ponts chimiques et physicochimiques et groupements fonctionnels impliqués, ainsi que la hiérarchisation des structures, de la particule et des molécules aux macro-agrégats.

La teneur et la nature des matières organiques sous la dépendance des pratiques culturales, jouent un rôle fondamental sur la stabilité structurale (formation-dégradation des agrégats). Ainsi, la teneur en matières organiques d'agrégats fins est bien corrélée à leur stabilité mesurée par la mouillabilité ou la dispersabilité de la fraction argileuse $<2 \mu \mathrm{m}$ (Chenu, 2001).

Bactéries et champignons interviennent dans ces phénomènes d'agrégation et structure des sols, par l'effet d'adhésion des produits du métabolisme bactérien ou racinaire dont la nature, considérée comme essentiellement polysaccharidique, doit être mieux définie. L'effet de l'enchevêtrement des particules de sol 
par les champignons filamenteux, comme le suggère la figure 8.3, doit aussi être mieux établie. Par ailleurs, la rôle de la faune (mécanique et biochimique) et en particulier de certains organismes « clés " (lombrics, termites...) dits aussi « organismes ingénieurs", leurs interactions avec les communautés microbiennes sont à faire progresser car vraiment peu connus (Lavelle et Spain, 2001).

Ces associations organominérales se manifestent aux échelles particulaires, cellulaires et moléculaires qui correspondent à leur structure de base et à celle des agrégats, comme l'illustrent les figures 8.2 à 8.4. On les observe aussi dans des sites ou micro niches particulières comme la rhizosphère.

\section{Interactions matières organiques - micro-organismes - minéraux dans les sols : quelques exemples et questions}

Divers types d'interactions entre constituants minéraux et matières organiques et entre matières organiques elles-mêmes se manifestent dans les sols. Ces interactions impliquent aussi les organismes du sol qui interviennent par leurs actions biochimiques et même physiques (mécanique dans le cas des vers de terre) visà-vis des constituants minéraux et organiques des sols (cf. ci-dessus et Berthelin et al., 1994 ; Lavelle et Spain, 2001). Il s'agit là d'un sujet bien vaste; aussi, à titre d'exemples, trois problèmes particuliers seront évoqués, mettant en jeu respectivement les argiles, les contaminants organiques et les éléments en traces métalliques.

\subsection{Exemples d'interactions " argiles - matières organiques - micro-organismes"}

Des expériences in vitro ont montré que la biodégradation et la minéralisation de composés organiques peut être, selon la nature de ces composés et la teneur et la nature d'argiles (kaolinite, montmorillonite), soit accélérée (cas de composés aldéhydiques, de l'acide urique), soit ralentie (cas d'acides gras aliphatiques, de protéines, de glucides, ....). Ces phénomènes sont à relier à des effets protecteurs des argiles par sorption des composés organiques (cf. complexes ou associations argilo-humiques) ou à des modifications de l'activité des micro-organismes. Diverses expériences indiquent que l'activité respiratoire peut être stimulée (cas de bactéries) ou ralentie (cas de champignons) par la présence de kaolinite ou de montmorillonite qui interviendraient, selon leur teneur, en modifiant la viscosité et la diffusion de l'oxygène, la disponibilité des substrats, la fixation de protons et la libération d'éléments minéraux nutritifs (Stotzky, 1986; 
Berthelin et al., 1994). Les argiles, et certaines d'entre elles en particulier (montmorillonite), favoriseraient la production microbienne de composés humiques ou de leurs précurseurs (composés phénoliques). Cet effet favorable des argiles sur l'humification s'observe aussi dans la rhizosphère des plantes, tant pour des systèmes racinaires associés ou non à des communautés microbiennes libres ou symbiotiques (Berthelin et al., 1994).

\subsection{Interactions entre " constituants organiques naturels - contaminants organiques - micro-organismes "; stabilisation à la biodégradation ("récalcitrante")}

Les processus de biotransformation, biodégradation, minéralisation des matières organiques des sols répondent à des besoins nutritionnels et énergétiques des micro-organismes, mais impliquent aussi des phénomènes de cométabolisme dans lesquels les substrats organiques ne sont pas utilisés pour fournir des nutriments et de l'énergie aux cellules microbiennes. Diverses revues récentes ont fait le point sur l'influence de la structure du sol sur la décomposition des composés organiques, sur l'implication de réactions biotiques et abiotiques, sur les interactions micro-organismes/argiles/enzymes, sur la survie des microorganismes. Un aspect important concerne le devenir des contaminants organiques et les interactions organiques-organiques.

L'incorporation de composés facilement dégradables, comme les acides aminés, dans des polymères de type humique va entraîner leur résistance (récalcitrance) à la biodégradation. Par exemple un acide aminé, la glycine (marquée au ${ }^{14} \mathrm{C}$ ), introduit dans un sol brun sous forme libre, sera pour $60 \%$ minéralisée en moins de dix jours. Si ce même composé est introduit dans le sol sous forme de polycondensat catéchol-glycine, il ne sera que faiblement minéralisé (moins de $10 \%$ ) pendant le même temps d'incubation et se retrouvera incorporé directement aux matériaux humiques stables. Les communautés microbiennes présentes ne dégradent alors que très lentement le polymère catéchol-glycine, qui est ainsi stabilisé. Sous forme de polycondensat alumineux, la dégradation sera encore plus ralentie.

Ces phénomènes de stabilisation de molécules facilement biodégradables s'observent aussi vis-à-vis de molécules xénobiotiques ou de leurs métabolites (Senesi et Miano, 1995 ; Reuter et al., 1999 ; Benoît et al., 2000 ; Barriuso et al., 2005).

Dans le cas d'un composé considéré comme un modèle de molécule xénobiotique, la 3-4 dichloroaniline (DCA), comparativement à son produit d'addition à l'orthoquinone (produit d'oxydation du catéchol) (DCPC), on observe des minéralisations dix à vingt fois supérieures pour le composé simple. Dans un sol 
lessivé, respectivement $6 \%$ et moins de $1 \%$ du DCA et du DCPC, sont minéralisés après 300 jours $d$ 'incubation. Dans ce cas des liaisons covalentes entre le DCA et l'orthoquinone seraient impliquées dans la formation de ce complexe organique-organique résistant à la biodégradation (Andreux et Munier-Lamy, 1994). Cette "récalcitrance " est accentuée par l'incorporation dans des matériaux humiques pas ou peu disponibles. Ce type de réaction illustre bien la formation de " résidus liés 》 stables (Barriuso et al., 2005) comme déjà indiqué ci-dessus.

Dans les deux cas, il peut s'ajouter l'absence ou l'insuffisance de microorganismes efficaces, la toxicité de ces polymères, leur stabilité et leur inaccessibilité. Toujours est-il que ces phénomènes et leur impact sur le devenir de contaminants ne sont pas encore bien définis.

\subsection{Impact des interactions micro-organismes - minéraux- matières organiques sur la mobilité des éléments en traces métalliques}

Les phénomènes qui guident les processus de mobilité des éléments en traces sont les conditions acido-basiques et d'oxydoréduction, la présence et la nature des ligands organiques et minéraux, la présence et la nature des collö̈des minéraux et organiques (matières organiques, argiles, oxyhydroxydes de fer, d'aluminium et de manganèse), la présence et l'activité des micro-organismes, les interactions et réactions aux interfaces " organismes-solutions-phases solides". Les situations sont souvent complexes et des phénomènes à caractère complémentaire et synergique ou bien à caractère opposé peuvent se manifester. Par exemple, la présence d'oxydes de fer augmentera la sorption des cations métalliques si le $\mathrm{pH}$ du sol augmente et celle des anions si le pH diminue. Cette fixation d'anions pourra aussi conduire à celle des cations par sorption spécifique. Ces deux phénomènes conduiront à la diminution de la mobilité des éléments en traces. Toutefois la diminution du $\mathrm{pH}$, si elle est suffisante, exercera un effet inverse en favorisant la solubilité des cations métalliques, ce qui sera le cas lors de la production d'acides minéraux (nitrique, sulfurique) ou organiques forts (oxalique, citrique) par les communautés microbiennes (bactériennes ou fongiques).

Le développement de conditions réductrices, suite à la consommation de l'oxygène du milieu par l'activité microbienne, conduira à la réduction bactérienne de $\mathrm{Fe} \mathrm{III}$ en Fe II, et donc à la dissolution des oxydes ferriques et celle des cations métalliques sorbés ou en substitution dans leur réseau cristallin. D'autres phénomènes, comme l'élévation du $\mathrm{pH}$ et des valeurs élevées, peuvent conduire : soit à la diminution (précipitation des cations sous forme de carbonates et hydroxydes, sorption des cations sur les oxydes, sorption des cations sur 
les substances humiques solides), soit à l'augmentation (complexation par des ligands solubles) de la mobilité des éléments métalliques en traces. Notons que cette mobilité est à appréhender non seulement vis-à-vis d'une potentielle lixiviation du sol mais surtout pour leur biodisponibilité vis-à-vis des végétaux et leur transfert vers la chaîne alimentaire. Il importe donc, dans de telles conditions de milieu et en présence de tels phénomènes, de bien définir les paramètres et de les hiérarchiser en précisant la réactivité des matières organiques.

\section{Conclusions, perspectives et recommandations}

Les matières organiques des milieux naturels et en particulier des sols mais aussi des eaux et sédiments méritent d'être mieux définies, même s'il ne s'agit pas d'établir des structures moléculaires d'acides humiques qui sont des molécules à structure " aléatoires 》, comme le soulignent aussi Balesdent et al., 2005. Très peu de composés, en effet, sont à structures moléculaires bien déterminées comme les molécules protéiques ou polysaccharidiques. Pour ces matières organiques correspondant aux substances humiques sensu lato, il s'agit de mieux déterminer à l'avenir :

1. leur stabilité et réactivité chimique et physicochimique comme la capacité de fixation et rétention et de modification de la biodisponibilité des métaux et non-métaux et de composés organiques en particulier de contaminants;

2. leur biodégradabilité, donc la nature des fractions biodégradables, source de carbone et d'énergie et de nutriments ;

3. leur rôle dans les transferts d'électrons pour les micro-organismes;

Il y a pour ces aspects un enjeu majeur, tant en ce qui concerne l'écologie microbienne et le fonctionnement des cycles biogéochimiques que la gestion des sols agricoles.

4. leur capacité de fixation, protection, conservation des protéines et des activités enzymatiques;

5. leur intérêt en tant que réserve de molécules ou structures moléculaires indicatrices des origines végétales ou microbiennes des matières organiques et des voies de biodégradation;

6. leurs voies de transformation et paramètres contrôlant leur stabilité (puits de carbone, structuration des sols) et leur biodégradabilité et évolution vers des formes organiques et/ou minérales (et leur implication vers la production de gaz à effet de serre, $\mathrm{CO}_{2}$ et $\mathrm{CH}_{4}$ en particulier); 
7. leur rôle en tant que source de nutriments calcium, magnésium, potassium, ..., mais aussi phosphore et soufre pour les micro-organismes, la faune du sol et les plantes;

8. leur forme et implication dans la stabilité et le transfert des associations organominérales (stabilité, dispersion, transfert de formes organominérales colloïdales particulaires...);

9. enfin, leur rôle et implications dans les couplages de cycles biogéochimiques (carbone-azote, carbone-phosphore, carbone-soufre).

Par ailleurs, d'un point de vue analytique, il y a lieu, d'un côté de caractériser les substances humiques en évitant les modifications, voire les dénaturations qui peuvent être liées aux méthodes préparatives (extraction, séparation...) et d'un autre, de croiser les voies d'approches.

Depuis quelques années on constate un renouveau des travaux de recherche, lié au développement et au couplage d'outils, aux approches interdisciplinaires et aux questions émergentes concernant les fonctions et usages des sols. En France, le Réseau "Matières organiques 》 a été réactivé, actualisé et élargi aux divers milieux et échelles où ces substances présentent de l'intérêt. Sa première réunion plénière s'est déroulée en janvier 2006. Il semble indispensable de soutenir ces réseaux et ces nouvelles démarches, ainsi que les travaux interdisciplinaires ou pluridisciplinaires qui se mettent en place.

\section{Encadré 8.2}

\section{Une équipe de chercheurs français a démonté le mécanisme qui favorise la formation des hydrocarbures}

\section{(d'après Caroline de Malet : « Découverte d'une nouvelle alchimie dans la formation du pétrole ", Le Figaro, 13 mai 2006)}

Une nouvelle étude parue le 12 mai 2006 dans la revue américaine Science, sous la signature de Pierre Albrecht, permet d'éclairer le processus de formation des hydrocarbures.

Tout comme le charbon, le pétrole, combustible fossile, se forme à travers un processus géologique qui remonte à des millions d'années. La houille de charbon s'est formée il y a $\mathbf{3 4 5}$ millions d'années, à l'époque carbonifère, dans un milieu marécageux, à partir de la décomposition des végétaux. Dans le cas du pétrole, ce sont des roches mères sédimentaires contenant 20 à $30 \%$ de carbone et déposées à une certaine profondeur d'eau, qui ont donné naissance aux hydrocarbures. 
Car, à partir d'une certaine profondeur, l'oxygène manque, ce qui est favorable à la préservation d'espèces carbonées. L'oxygène, au contraire, entraîne leur dégradation et leur transformation en gaz carbonique par des processus microbiens et des bactéries, sur une période de temps étalée entre 10000 et 100000 ans. Il s'agit donc d'une énergie fossile qui échappe donc au cycle normal du carbone.

C'est grâce à cette étape préalable de préservation que des matières carbonées se sont accumulées dans le sous-sol pour donner naissance aux sources d'énergie fossile telles que pétrole ou gaz. Une fois le sédiment formé, la présence d'eau combinée à une hausse de température à une grande profondeur ( 2 ou 3 kilomètres) entraîne leur formation, processus étalé sur plusieurs millions d'années. Ce phénomène de la préservation est bien connu des scientifiques dans son principe, mais ses mécanismes sont encore mal expliqués.

Une équipe de géochimistes français du laboratoire de géochimie bioorganique de Strasbourg, associée au Geologisches Institut de Zurich, vient de montrer dans celte publication que " contrairement à un dogme largement accepté, les bactéries ne sont pas directement impliquées dans ce processus de conservation », comme l'explique Pierre Albrecht, l'un des membres de cette équipe.

Il ne s'agit pas d'un processus biologique, mais d'un mécanisme à mettre sur le compte des substances soufrées produites par les bactéries a partir des sulfates. C'est ainsi que des produits chimiques instables vont réagir ef se transformer en produits stables fossiles.

\section{Références bibliographiques}

Adrian Ph., Andreux F., Metche M., Mansour M. et Korte FG. (1986). Autoxydation des ortho-diphenols catalysés par les ions $\mathrm{Fe}^{2+}$ et $\mathrm{Mn}^{2+}$ : un modèle de formation des acides humiques. CR Acad Sci Paris, 303 II, 17 : 1615-1618.

Amiri F., Bornick H. et Worch E. (2005). Sorption of phenols onto sandy aquifer material : The effect of dissolved organic matter (dom). Water Research, 39 (5) :933-941.

Andreux F. et Munier-Lamy C. (1994). Genèse et propriétés des molécules humiques. Ch. 6. In Bonneau M. et Souchier B. (eds). Pédologie 2. Constituants et propriétés du sol. Masson, Paris, 109-142.

Andreux F. (1996). Humus in world soils. In Piccolo A. (ed). Humic substances in terrestrial Ecosystems. Elsevier Science, Amsterdam, p. 45-100. 
Balesdent J., Arrouays D., Chenu C. et Feller C. (2005). Stockage et recyclage du carbone. In Girard MC., Walter C., Rémy JC., Berthelin J., Morel JL. (eds). Sols et Environnements. Paris, Dunod, 37 : 238-259.

Barriuso E., Benoit P., Charnay MP., Coquet Y., Louchart X., Schiavon M., Aurousseau $P$. (2005). Pollutions diffuses : mobilité et persistance des polluants organiques dans les sols. In Girard MC., Walter C., Rémy JC., Berthelin J., Morel JL. (eds). Sols et Environnements. Paris, Dunod, 37 : 408-425.

Benedetti M., Ranville JF., Ponthieu M. et Pinheiro JP. (2002). Field-flow fractionation characterization and binding properties of particulate and colloidal organic matter from the Rio Amazon and Rio Negro. Organic Geochemistry, $33(3): 269-279$.

Benoît P., Barriuso E., Bergheaud V. et Etievant V. (2000). Binding capacities of different soil size fractions in the formation of herbicide-bound residues. Agronomie, 20 (5) : 505-512.

Berthelin J., Andreux F., Tessier D. (2005). Constituants originaux du sol : réactivité et interactions. In Girard MC., Walter C., Rémy JC., Berthelin J., Morel JL. (eds). Sols et Environnements. Paris, Dunod, 37 : 34-52.

Berthelin J., Leyval C. et Toutain F. (1994). Biologie des sols. Rôle des organismes dans l'altération et l'humification. Ch. 7. In Bonneau M. et Souchier B. (eds). Pédologie 2. Constituants et propriétés du sol. Masson, Paris, 143-237.

Berthelin J., Munier-Lamy C., Portal JM., Toutain F. (1999). Physico-chemical characterisation, reactivity and biodegradability of soil natural organic matter. In Baveye Ph., Block JC., Goncharuk VV. (eds). Bioavailability of organic xenobiotics in the environment. Kluwer Academic Publisher, Dordrecht, 251 296.

Bruckert S. (1994). Analyse des complexes organo-minéraux des sols. p. $275-$ 295. In Bonneau M. et Souchier B. (eds). Pédologie 2. Constituants et propriétés du sol. Masson, Paris.

Chenu C. (2001). Le complexe argilo-humique des sols : état des connaissances actuelles. C. R. Acad. Agric. Fr., 87 : 3-12.

Dommergues Y. et Mangenot F. (1970). Écologie microbienne du sol. Masson, Paris, $796 \mathrm{p}$.

Dudal Y. et Gérard F. (2004). Accounting for natural organic matter in aqueous chemical equilibrium models: a review of the theories and applications. Earth-Science Reviews, 66 : 199-216.

Gobat JM., Aragno M. et Mathey W. (2003). Le sol vivant. Base de Pédologie Biologie des sols. Presses Polytechniques et Universitaires Romandes. EPFL, Lausanne, $568 \mathrm{p}$. 
Hayes MHB., McCarthy P., Malcolm RL. et Swift R. (eds). (1989). Humic Substances II. In: Search of Structure. John Wiley and Sons, Chichester.

Hebting Y., Schaeffer P., Behrens A., Adam P., Schmitt G., Schneckenburger P., Bernasconi SM. et Albrecht P. (2006). Biomarker evidence for a major preservation pathway of sedimentary organic carbon. Science, 312 : 1627 1631.

Kögel-Knabner I. (2000). Analytical approaches for characterizing soil organic matter. Organic Geochemistry, 31 : 609-625.

Kujawinski EB. (2002). Electrospray lonization Fourier Transform lon Cyclotron Resonance Mass Spectrometry (ES IFT-ICR MS) : Characterization of complex environmental mixtures. Environmemtal Forensics, 3 : 207-216.

Kujawinski EB., Del Vecchio R., Blough NV., Klein GC. et Marshall AG. (2004). Probing Molecular-level transformations of dissolved organic matter : Insights on photochemical degradation and protozoan modification of DOM from Electrospray lonization Fourier Transform Ion Cyclotron Resonance Mass Spectrometry. Marine Chemistry, 92 ; 23-37.

Lamy I., Sterckeman T., Cambier P., Jaffrezic A., Van Oort F., Baize D., Chaussod R., Denaix L. et Cornu S. (2005). Présence et impact des éléments en traces dans les sols. In Girard MC., Walter C., Rémy JC., Berthelin J., Morel JL. (eds). Sols et Environnements. Dunod, Paris, 37 : 469-487.

Lavelle P. et Spain AV. (2001). Soil Ecology, Kluwer Academic Press, Dordrecht, $654 \mathrm{p}$.

Marschner B. et Kalbitz K. (2003). Controls of bioavailability and biodegradability of dissolved organic matter in soils. Geoderma, 113 (3-4) : 21 1-235.

Mc Bride M. (1994). Environmental chemistry of soils. Oxford University Press, New York, $406 p$.

Paul EA. et Huang PM. (1980). Chemical aspects of Soils. In O. Hutzinger (ed). Environmental Chemistry, Volume 1, Part A., The Natural Environment and the Biogeochemical Cycles. Springer Verlag, 69-86.

Quiquampoix H. (2000). Mechanisms of protein adsorption on surfaces and consequences for soil extracellular enzyme activity. In Bollag JM. et Stotzky G. (eds). Soil Biochemistry. Marcel Dekker, New York, 171-206.

Reuter S., Ilim M., Munch JC., Andreux F. et Scheunert I. (1999). A model for the formation and degradation of bound residues of the herbicide ${ }^{14} \mathrm{C}$ isoproturon in soil. Chemosphere, 39 (4) : 677-639. 
Schnitzer M., 1985. Nature of nitrogen in humic substances 303-325. In Aiken GR., McKnight DM., Wershaw RL., MacCarthy P. (eds). Humic Substances in Soil, Sediment and Water. John Wiley and Sons, New York.

Senesi N. et Miano TM. (1995). The role of abiotic interactions with humic substances on the environmental impact of organic pollutants. In Huang PM., Berthelin J., Bollag JM., McGill WB. (eds). Environmental Impacts of Soil Components Interactions. Vol. I. Natural and Anthropogenic Organics, page A., Lewis Publishers, Chelsea, Ml, 31 1-335.

Stotzky G. (1986). Influence of soil mineral colloïds on metabolic processes, growth, adhesion and ecology of microbes and viruses. p. 305-428. In Huang PM. et Schnitzer M. (eds). Interactions of soil minerals with natural organics and microbes. SSSA Special Publication n 17, Madison, USA, $606 \mathrm{p}$.

Vrdoljak G. et Sposito G. (2002). Soil aggregate hierarchy in a brasilian oxisol. In Violante A, Huang PM., Bollag JM. et Gianfreda L. (eds). Soil Mineral Organic matter - Microorganism interactions and ecosystem health. Elsevier p. 197-217.

Zsolnay A. (2003). Dissolved organic matter : artefacts, definitions, and functions. Geoderma, $113(3-4)$ : 187-209. 
